Preprint typeset in JHEP style - HYPER VERSION

ArXiv:0707.1324

CPHT-RR027.0507

\title{
Exploring improved holographic theories for QCD: Part I
}

\author{
U. Gürsoy ${ }^{1,2}$, E. Kiritsis ${ }^{1,3}$ \\ 1 CPHT, Ecole Polytechnique, CNRS, 91128, Palaiseau, France \\ ( UMR du CNRS 7644). \\ $\sqrt[2]{\text { Laboratoire de Physique Théorique, }}$ \\ Ecole Normale Supérieure, 24, Rue Lhomond, Paris 75005, France. \\ $\sqrt[3]{\text { Department of Physics, University of Crete, } 71003 \text { Heraklion, Greec }}$
}

\begin{abstract}
Various holographic approaches to QCD in five dimensions are explored using input both from the putative five-dimensional non-critical string theory as well as QCD. It is argued that a gravity theory in five dimensions coupled to a dilaton and an axion may capture the important qualitative features of pure YM theory. A part of the effects of higher $\alpha^{\prime}$-corrections is resummed into a dilaton potential. The potential is shown to be in one-to-one correspondence with the exact $\beta$-function of QCD, and its knowledge determines the full structure of the vacuum solution. The geometry near the UV boundary is that of $\mathrm{AdS}_{5}$ with logarithmic corrections reflecting the asymptotic freedom of QCD. We find that all relevant confining backgrounds have an IR singularity of the "good" kind that allows unambiguous spectrum computations. Near the singularity the 't Hooft coupling is driven to infinity. Asymptotically linear glueball masses can also be achieved. The classification of all confining asymptotics, the associated glueball spectra and meson dynamics are addressed in a companion paper ArXiv:0707.1349
\end{abstract}

KEYwORDs: AdS/CFT, holography, gauge theory, QCD, Large-N limit. 


\section{Contents}

1. Introduction and conclusions 2

2. Motivating the gravitational dual of a large- $\mathrm{N}_{c}$ gauge theory 13

2.1 The spectrum 13

2.2 The associated branes 15

2.3 The effective action 15

3. Holographic large $\mathrm{N}_{c} \mathrm{YM}$ : the vacuum structure 21

3.1 General geometry and the geometric invariants 21

3.2 The phase variable $X$ and the $\beta$-function 23

4. Asymptotics of the geometry 24

4.1 Asymptotic freedom and the UV geometry 24

4.2 The $\alpha^{\prime}$ corrections 25

4.3 The UV geometry 26

4.4 Confinement and the IR geometry 27

5. Examples of background geometries 29

5.1 Standard QCD type two-loop $\beta$-function 29

$5.2 \beta$-function with an IR fixed point 31

6. Fluctuations near the boundary 34

6.1 Dilaton fluctuations 35

6.2 Axion fluctuations 36

Acknowledgments 36

Appendices 38

A. Asymptotic expansions in the (UV) weak-coupling regime 38

A.1 Scalar curvature invariants in the UV regime 41

A.2 The two-loop $\beta$-function coefficients of pure gauge theory 42

B. General potentials at string tree level 42

B.1 Corrections to the axion terms

B.2 Corrections to the gauge coupling constant identification 47

B.3 Other higher derivative corrections at the tree level 47 
C. Perturbative analysis near an extremal (AdS) point of the dilaton potential

D. Analysis of the dilaton potential with single exponential 50

D.1 The $X=1$ fixed point 51

D.2 The $X=-1$ fixed point 51

D.3 The $X=-\frac{1}{a}$ fixed point 52

D.4 Flow solutions 52

D.4.1 The solution: $-1<X<-1 / a$

D.4.2 The solution: $-1 / a<X<+1$

E. $\beta$-function with an exponential tail [54

References 56

\section{Introduction and conclusions}

The correspondence between large- $N_{c}$ gauge theory and string theory first advocated by 't Hooft [1] has taken a novel (and mostly unexpected) form after the Maldacena conjecture, [2]. Although the most precise version of the correspondence was formulated for the (conformally invariant) maximally supersymmetric Yang Mills theory in four dimensions, in several other cases, holographic techniques proved useful, and several gravitational duals describing confining theories in the IR have been proposed. One concrete application of holographic duality is to establish a novel quantitative understanding of QCD, in particular of phenomena where strong IR physics is important. These include confinement, chiral symmetry breaking, as well as quantitative issues about the meson and baryon spectrum and their interactions.

Critical holographic models obtained as solutions of ten-dimensional string theory [3, 5, 6] have been successful in their qualitative description of confinement and other IR dynamics, including a semi-quantitative agreement of the glueball spectrum [0] and thermal properties [3]. At the same time, the theories contain Kaluza-Klein modes, not expected in QCD, with KK masses of the same order as the dynamical scale of the gauge theory. Above this scale the theories deviate from QCD. In the solution associated to non-supersymmetric wrapped $D_{4}$ branes, [3] the UV completion should be thought of as a 6D theory on M5 branes. In the Chamsedinne-Volkov solution [4], interpreted holographically by Maldacena and Nunez, [5], the theory becomes the $6 \mathrm{~d}$ theory obtained by wrapping $\mathrm{NS}_{5}$ branes on a two-sphere. Finally in the Klebanov-Strassler solution, [6] the theory passes through a large set of Seiberg dualities to end up with a non-conformal quiver theory in the UV. 
An obvious way to avoid the extra KK modes is to consider non-critical string theories. Indeed, if our general intuition about holography is correct, QCD should be described by a string theory living in 5 dimensions. However, it is expected from general arguments that non-critical string duals of large- $N_{c}$ gauge theories will have background curvatures and other invariants of the same order as the string scale and an $\alpha^{\prime}$ expansion is not expected to be a priori a reliable approximation. Such an expectation is partly based on the fact that the part of the potential due to the central charge deficit is of order $\mathcal{O}(1)$ in $\ell_{s}^{-2}$ units. One may also give a more rigorous statement [8] for supersymmetric theories, based on the conformal anomaly [9].

Despite the hostile environment of non-critical theory, several attempts have been made to understand holographic physics in this regime, based on two-derivative gravitational actions, [10, 11, 8, 12. Moreover, such attempts were seconded by studies of the physics of probe branes in non-critical backgrounds [13, 14, 15] and noncritical orientifolds [16] that provide side information on the holographic approaches. Most of these attempts focused on $4 \mathrm{~d}$ CFTs with $\mathcal{N}=1$ supersymmetry [11, 8] or without it [10, 8, 12]. The rational has been, that although $\alpha^{\prime}$ corrections are generically expected to be substantial, qualitative information should be (mostly) reliable. Moreover, the high symmetry may guarantee that some quantities could be reliably computed. Part of this intuition stems from exact solutions to $2 \mathrm{~d}$ WZW CFTs where ratios of conformal weights can be calculated classically, and are exact to all orders in the $\alpha^{\prime}$ expansion.

A different and more phenomenological approach was in the meantime developed, and is now known as AdS/QCD. The original idea was formulated in [17] and it was successfully applied to the meson sector in [18, 19]. The bulk gravitational background consists of a slice of $A d S_{5}$, and a constant dilaton. There is a UV and an IR cutoff. Moreover, the confining IR physics is imposed by boundary conditions at the IR boundary. This approach seems very crude and indeed it is, when applied to the pure glue sector. However it has been partly successful in studying meson physics despite the fact that the dynamics driving chiral symmetry breaking must be imposed by hand via IR boundary conditions.

In this paper and its companion [20], we will investigate a mostly phenomenological approach that runs somewhere in-between non-critical string theory approaches and AdS/QCD. On the one hand we will investigate and motivate what kind of effective theory we expect to describe QCD, based on our understanding of string theory. On the other hand we would like to match this with what we expect from QCD in the UV and the IR.

There is intriguing evidence from studies of QCD, that high-dimension operators, that should be associated to stringy modes, are not very important for (some of the) physics at short distances. This is suggested by the success of SVZ sum rules, 21] which tie together the UV physics with the IR physics in QCD. A counter-argument relies on the fact that as we understand from critical holography, the large 't Hooft 
coupling limit $\lambda \gg 1$ is necessary to suppress higher $\alpha^{\prime}$ corrections. Therefore, in QCD, as this coupling is driven to zero in the UV, such corrections will become dominant. However, as we argue below, progress can be achieved in this direction despite the difficulties.

Pure $4 \mathrm{~d}$ YM at large $N_{c}$ is expected to be dual to a string theory living in 5 dimensions. The relevant low-lying fields are expected to be dual to the lowest dimension operators, namely the graviton (dual to $T_{\mu \nu} \sim \operatorname{Tr}\left[F_{\mu \nu}^{2}-\frac{1}{4} \delta_{\mu \nu} F^{2}\right]$ ), the dilaton $\phi$ (dual to $\operatorname{Tr}\left[F^{2}\right]$ ) and the $\mathrm{RR}$ axion $a$ (dual to $\operatorname{Tr}[F \wedge F]$ ). Moreover, in the string frame, the theory has a very simple dilaton potential $\sim \delta c$ that reflects the fact that the associated string theory is non-critical. The theory however must contain a RR four-form whose flux seeds the $D_{3}$ branes and therefore generates the $U\left(N_{c}\right)$ gauge group. The presence of such a form is compatible with the spectrum of operators we advocate, as in 5 dimensions a four-form is non-propagating. By integrating out the four-form we generate new terms in the dilaton potential. This is shown in section 2 and appendix B. Therefore, taking into account higher $\alpha^{\prime}$ terms involving the four-form only, generates a non-trivial potential for the dilaton $\phi$. Hence, the special nature of the four-form implies that its field strength, although it carries powers of $\alpha^{\prime}$, effectively carries no derivatives, and it should therefore be incorporated in the potential.

There are two properties that this potential satisfies. The first is that it is a nontrivial function of the 't Hooft coupling $\lambda \sim N_{c} e^{\phi}$, ( $\lambda$ thus defined, is expected to remain finite in the large- $N_{c}$ limit). The second is that (a) it has a regular expansion around $\lambda=0$ (b) it vanishes as $\lambda^{\frac{4}{3}}$ at $\lambda=0$ (in the Einstein frame). To next to leading order we expect

$$
V(\lambda)=\lambda^{\frac{4}{3}}\left(\delta c-\lambda^{2}+\cdots\right)
$$

This potential was established in [8] and is generic in dimensions 5, 4 and 3. The fact that it vanishes at $\lambda=0$ implies that the asymptotic geometry in the UV does not approach $\mathrm{AdS}_{5}$, as we might expect. This is analyzed in detail in appendix $\mathrm{D}$. However the potential above has another intriguing property: it has an AdS minimum at a finite value of the 't Hooft coupling, and therefore there is a related $\mathrm{AdS}_{5}$ solution with fixed finite 't Hooft coupling. The expectation that this $\mathrm{AdS}_{5}$ may be the UV limit of a non-trivial solution with (logarithmically) running $\lambda$ seems however to fail as explained in appendix Q. Therefore the only "correct" weak-coupling asymptotics of the dilaton potential must satisfy

$$
\lim _{\lambda \rightarrow 0} V(\lambda)=V_{0} \neq 0
$$

This guarantees the existence of a solution which is asymptotically $\mathrm{AdS}_{5}$ near the UV boundary, provided the 't Hooft coupling vanishes there. Moreover, if we wish that in the same UV region, the 't Hooft coupling runs logarithmically with energy, 
then the weak coupling expansion of the potential must be of the form

$$
V(\lambda)=V_{0}\left(1+\sum_{n=1}^{\infty} V_{n}\left(\lambda^{a}\right)^{n}\right)
$$

with $a$ some positive real number that can be shifted to the wave function renormalization of the dilaton. As suggested by the perturbative QCD $\beta$-function we will select $a=1$. As we show in section 2 and appendix $\mathrm{A}$, this expansion of the potential is equivalent to the perturbative expansion of the QCD $\beta$-function and the coefficients $V_{n}$ can be directly related to the perturbative $\beta$-function coefficients.

There are two obvious questions that accompany the discussion above:

1. What is the origin of the non-zero constant $V_{0}$ ?

2. In the arguments above we have neglected higher $\alpha^{\prime}$-corrections involving the curvature and derivatives of the scalars.

As we indicate in appendix B.3, a non-zero constant $V_{0}$ in the effective dilaton potential may be generated from the higher-curvature corrections. Although this also implies that higher $\alpha^{\prime}$-corrections cannot be neglected after all, we would like to take the bold step and assume that the only quantitatively important part of this class of $\alpha^{\prime}$ corrections is just to generate a non-zero $V_{0}$ as well as the rest of the terms of the dilaton potential. This step, has as a consequence that we will eventually deal with a two-derivative effective action but with a general dilaton potential satisfying (1.3) at weak 't Hooft coupling. Although this approach cannot be rigorously defended, our attitude is exploratory. An extra motivation comes from the success of the SVZ sum rules [21]. Moreover, as we show below, our results are encouraging.

To summarize the discussion above, our starting point is an action (in the Einstein frame) of the form

$$
S=M^{3} N_{c}^{2} \int d^{5} x \sqrt{g}\left[R-\frac{4}{3} \frac{(\partial \lambda)^{2}}{\lambda^{2}}-\frac{Z(\lambda)}{2 N_{c}^{2}}(\partial a)^{2}+V(\lambda)\right]
$$

with the potential $V(\lambda)$ having around $\lambda=0$ an asymptotic expansion of the form (1.3).

The axion kinetic term in (1.4) deserves some comments. In a way similar with the potential, we expect a non-trivial function of the dilaton $Z(\lambda)$ multiplying its kinetic term. ${ }^{1}$ Moreover, as explained in detail in appendix B.1, the axion kinetic term is of order $\mathcal{O}\left(1 / N_{c}^{2}\right)$ compared with the rest of the terms in (1.4). This is due in string theory to the fact that the axion is a RR field and therefore has a suppressed dilaton dependence. ${ }^{2}$ The same argument indicates that terms involving

\footnotetext{
${ }^{1} \mathrm{~A}$ priori, similar functions might also multiply the Einstein term and the dilaton kinetic term. Such terms can however be removed by Weyl-rescaling of the metric and redefining $\lambda$.

${ }^{2}$ This is not valid for the RR four-form as its field strength is linear in the number of colors $N_{c}$.
} 
higher powers of $(\partial a)^{2}$ will be further suppressed at large $N_{c}$. Therefore, the $\alpha^{\prime}-$ expansion of the axion terms is effectively an $1 / N_{c}$ expansion. The perturbative asymptotics of $Z(\lambda)$ therefore should be

$$
Z(\lambda)=Z_{a}+\mathcal{O}\left(\lambda^{2}\right) \quad, \quad \lambda \rightarrow 0
$$

String theory gives a $\lambda^{2}$ contribution to leading order. However as in the case of the potential, higher derivative corrections are expected to generate also a constant piece. This turns out to be in agreement with perturbative QCD.

As shown in section 4 of [20], $Z(\lambda)$ determines an analogue of a $\beta$-function for the QCD $\theta$-parameter. This interpretation has however caveats that are discussed in the same section. It is obvious that while searching for the solution of the equations of motion stemming from the action (1.4), that will describe the QCD vacuum, the axion contribution can be neglected to leading order in $1 / N_{c}$. Once this solution is found, the axion equation of motion can be solved in order to determine the profile of the QCD $\theta$-parameter and its associated physics.

Therefore, to leading order in $1 / N_{c}$, the vacuum structure of $\mathrm{QCD}$, is captured by a solution of (1.4) with the appropriate $A d S_{5}$ asymptotics. All the properties of the $4 \mathrm{D}$ gauge theory depend on a single function of the 't Hooft coupling, the superpotential $W(\lambda)$, defined $\mathrm{as}^{3}$ :

$$
V(\lambda)=-\frac{4}{3} \lambda^{2}\left(\frac{d W}{d \lambda}\right)^{2}+\frac{64}{27} W^{2} .
$$

This dependence can be mapped (up to the overall AdS length $\ell$, related to $V_{0}$ by $\left.V_{0}=\frac{12}{\ell^{2}}\right)$ to the (exact) $\beta$-function of $\mathrm{QCD}, \beta(\lambda)$. In particular, for small $\lambda$, all coefficients of the superpotential can be mapped to the perturbative terms of the $\beta$-function. Concretely, the following relation holds:

$$
\beta(\lambda)=-\frac{9}{4} \lambda^{2} \frac{d \log W(\lambda)}{d \lambda}
$$

Moreover fitting to QCD data we learn that, [20]

$$
\ell \simeq 6.26 \ell_{s}
$$

We expect three integration constants for the equations of motion of the metric and $\lambda$. One of them fixes $W(\lambda)$ as a solution of eq. (11.6). We show in [20 that this integration constant is completely fixed by using asymptotic freedom as an input from the gauge theory. Among the second and third integration constants that arise

\footnotetext{
${ }^{3}$ Generically, passing from the potential to the superpotential and the associated first order equations involves an arbitrary constant that can be thought of as the single constant of integration of (1.6). However in our case it turns out that the relevant solution is special, and does not have this extra dependence on an arbitrary constant. This is explained in appendix $\mathrm{E}$ of [20].
} 
from the first-order differential equations for $\lambda$ and $A$, only a single combination remains due to the reparametrization invariance. This remaining integration constant amounts to a definition of $\Lambda_{Q C D}$. ¿From eq. (11.7) it is apparent that the exact $\beta$ function determines completely the geometry up to a choice of $\Lambda_{Q C D}$.

One result, in our two-derivative approach, concerns the investigation of potential confining backgrounds in the IR. We choose the conformal coordinate system and write the Einstein metric as

$$
d s^{2}=e^{2 A(r)}\left(d r^{2}+\eta_{\mu \nu} d x^{\mu} d x^{\mu}\right)
$$

with the $\mathrm{AdS}_{5}$ boundary at $r=0$. As we show, $e^{A(r)}$ monotonically decreases from $\infty$ at the UV boundary, to 0 , or to a finite value, in the IR (see section 3.2). We therefore take the scale factor in the Einstein frame as our definition for the energy of the gauge theory.

$$
E=e^{A(r)}
$$

In the case when the Einstein frame scale factor remains finite at the IR singularity, this indicates that the dual theory is defined only above a certain energy. Such models however fail to satisfy some of the properties that are believed to hold in confining theories, namely the screening of the magnetic color charges.

In our analysis of the non-perturbative regime, we give a general classification of possible IR geometries according to their confining properties. We use, as a characterization of confinement, the Wilson loop area law: by "confining," we label those backgrounds for which the QCD string (identified with the fundamental string) has a finite tension. We then analyze various properties of confining backgrounds. Here is a summary of our findings:

- We provide a relation between the $\beta$-function of the gauge theory defined by an infinite series expansion in the 't Hooft coupling, and the scalar potential (or rather the superpotential) of the gravitation theory defined by a similar expansion in the dilaton.

- We study certain $\alpha^{\prime}$ corrections to the scalar potential. In particular, we find that in the identification of the $\beta$-function and scalar potential coefficients, the first two scheme-independent coefficients of the $\beta$-function receive no $\alpha^{\prime}$ corrections. Moreover we find that the $\alpha^{\prime}$ corrections to the identification of the energy scale with the scale factor of the metric can be set to zero in a particular scheme for computing the higher order $\beta$-function coefficients, $b_{2}, b_{3}, \ldots$.

- We show that all confining backgrounds have a singularity in the Einstein metric at some value $r_{0}$ of the $r$ coordinate. There are two distinct cases for the position $r_{0}$ of the IR singularity. One possibility is finite $r_{0}$. The other is $r_{0}=\infty$. The IR singularity is always of the "good kind" [22]: fluctuation 
spectra of various fields are well defined and are not affected directly by the presence of the singularity.

- For regular dilaton potentials ${ }^{4}$, the 't Hooft coupling $\lambda$ always becomes infinite at the IR singularity.

- In the case $r_{0}=\infty$, the string frame metric is not only regular at the IR singularity but its curvature also vanishes. Put otherwise, in the string frame, the IR singularity is only due to the diverging 't Hooft coupling constant. This suggests that the supergravity approximation may be a good approximation in the IR region.

- We classify all superpotentials $W(\lambda)$ that give rise to confining backgrounds: We parametrize their asymptotics for $\lambda \rightarrow \infty \mathrm{as}^{5}$ :

$$
W(\lambda) \sim(\log \lambda)^{\frac{P}{2}} \lambda^{Q} \quad, \quad P, Q \in R .
$$

The 't Hooft couplings diverges in the IR as

$$
\lambda \sim E^{-\frac{9}{4} Q}\left(\log \frac{1}{E}\right)^{\frac{P}{2 Q}}, \quad E \rightarrow 0 .
$$

1. $Q>2 / 3$ or $Q=2 / 3$ and $P>1$ leads to confinement and a singularity at finite $r=r_{0}$. The scale factor $e^{A}$ vanishes there as

$$
e^{A}(r) \sim\left\{\begin{array}{ll}
\left(r_{0}-r\right)^{\frac{4}{9 Q^{2}-4}} & Q>\frac{2}{3} \\
\exp \left[-\frac{C}{\left(r_{0}-r\right)^{1 /(P-1)}}\right] & Q=\frac{2}{3}
\end{array},\right.
$$

where $C$ is a positive constant related to the integration constants.

2. $Q=2 / 3$, and $0 \leq P<1$ leads to confinement and a singularity at $r=\infty$ The scale factor $e^{A}$ vanishes there as

$$
e^{A}(r) \sim \exp \left[-C r^{1 /(1-P)}\right]
$$

3. $Q=2 / 3, P=1$ leads to confinement but the singularity may be at a finite or infinite value of $r$ depending on subleading asymptotics of the superpotential.

The above exhaust all cases that confine. All other cases $(Q<2 / 3$, or $Q=2 / 3$ and $P<0$ ) fail the Wilson loop test. By eq. (1.7), this classification directly relates the confining property to the IR behavior of the QCD $\beta$-function.

\footnotetext{
${ }^{4} \mathrm{We}$ assume that the potential, hence the $\beta$-function, do not have singularities at finite $\lambda$.

${ }^{5}$ Since our results are continuous in the parameters $P$ and $Q$, our classification also extends to any functions $W(\lambda)$ that has a well-defined limit for $\lambda \rightarrow \infty$.
} 
- If $Q<2 \sqrt{2} / 3$, no ad hoc boundary conditions are needed but UV and IR normalizability completely determines the glueball spectrum. This is unlike standard AdS/QCD and other approaches. Since the spectrum is completely determined from the geometry, and, as discussed above, the latter is in one-toone correspondence with the $\beta$-function, our construction provides a direct link between the $\beta$-function and the spectrum. On the other hand, when $Q>2 \sqrt{2} / 3$, the spectrum is not well defined without extra boundary conditions in the IR because both solutions to the mass eigenvalue equation are IR normalizable. ${ }^{6}$

- For all potentials that confine, the spectrum of $0^{++}$and $2^{++}$glueballs has a mass gap. Moreover, except for the borderline case $Q=2 / 3, P=0^{7}$, the spectrum is also purely discrete. For the $0^{+-}$glueballs an extra assumption is needed about the strong-coupling asymptotics of the function $Z(\lambda)$ in (1.4):

$$
Z(\lambda) \sim \lambda^{d} \quad, \quad d>2 \quad \text { as } \quad \lambda \rightarrow \infty
$$

If this is satisfied, the $0^{-+}$spectrum is also gapped and discrete. We find that in $\mathrm{QCD} d=4$.

- In all the physically interesting confining backgrounds (i.e. those that do not require extra boundary conditions in the IR), the magnetic color charges are screened. This is shown by studying the $\mathrm{D}_{1}$ branes embedded in the geometry, see [20]. This is an improvement with respect to AdS/QCD models, where magnetic quarks are also confined instead of being screened.

- Of all the possible confining asymptotics, there is a unique one that guarantees "linear confinement" for all glueballs. It corresponds to the case $Q=2 / 3, P=$ $1 / 2$, i.e. strong-coupling superpotential and $\beta$-function asymptotics:

$$
W(\lambda) \sim(\log \lambda)^{\frac{1}{4}} \lambda^{\frac{2}{3}} \quad, \quad \beta(\lambda)=-\frac{3}{2} \lambda\left[1+\frac{3}{8 \log \lambda}+\cdots\right]
$$

In this case the 't Hooft coupling diverges with energy as

$$
\lambda \sim E^{-\frac{3}{2}}\left(\log \frac{1}{E}\right)^{\frac{3}{8}}
$$

in the IR. This choice also seems to be preferred from considerations of the meson sector as discussed below.

\footnotetext{
${ }^{6}$ In [12] an exact solution was studied based on the potential $W(\lambda)=W_{0}+W_{1} \lambda^{\frac{4}{3}}$ in our normalizations. This generates logarithmic running in the UV and corresponds to a confining background in the IR. However, as it corresponds to $Q=4 / 3$, extra boundary conditions are needed at the IR singularity. The spectrum heavily depends on the choice of these boundary conditions.

${ }^{7}$ This is also exactly the linear dilaton background in the IR. The spectrum of glueballs is however continuous in this case. Because of this, this case is considered no further.
} 
- The meson sector, assuming $N_{f} \ll N_{c}$, is implemented by using $N_{f}$ pairs of $D_{4}-\bar{D}_{4}$ branes embedded in the 5-dimensional background along the lines first pointed-out in ten dimensions in [23]. Moreover, the open string tachyon field is included and it is dual to the scalar and pseudoscalar quark bilinears as first advocated in [24]. In section 5 of [20] we have studied the non-linear equation that determines the tachyon profile (vev). It was shown in [24] that consistency with the anomaly structure implies that the tachyon field must diverge before or at the IR end of space. What we find here is that for the confining background the tachyon necessarily diverges at the IR singularity, signaling chiral symmetry breaking and the IR recombination of the flavor branes. Moreover, it is found that although the tachyon cannot diverge before the IR singularity, its derivatives do generically diverge. Such a divergence is physically unacceptable. Imposing its absence, determines the quark vacuum condensate in terms of the UV quark masses that act as sources for the tachyon field.

- As in [24], the spectrum of mesons exhibits (almost) linear confinement due to the tachyon potential rather that the graviton-dilaton background. We have also studied the masses of the simplest mesons, the vectorial ones which are independent of the details of chiral symmetry breaking. They depend in general on a combination of the confining QCD scale (appearing in the graviton-dilaton data) and the AdS length $\ell$. We find that the special background advocated above, corresponding to $P=1 / 2$, which provides linear confinement in the glueball sector is also the one in which meson masses do not depend on the $\mathrm{AdS}_{5}$ scale, as expected on general grounds. Therefore we obtain a simple linear relation between the mass scales of glueballs and generic mesons (this does not include the pseudo-Goldstone bosons).

- We calculate numerically the $0^{++}, 0^{-+}$and $2^{++}$glueball spectra for $\beta$-functions that interpolate between the standard perturbative QCD regime and the confining regime, both for the $r_{0}$ finite and infinite cases. We compare the glueball spectra with lattice results. Although there is no universal consensus on the reliability of various lattice results and their relationship to the large- $N_{c}$ limit, we attempt a comparison with various confining IR asymptotics. We find that the cases of $r_{0}=\infty$ are preferred by the data.

- We analyze the axion sector of the theory in section 4 of [20]. We solve the equation for the axion to find

$$
a(r)=\left(\theta_{U V}+2 \pi k\right) \frac{\int_{r}^{r_{0}} \frac{d r}{e^{3 A} Z(\lambda)}}{\int_{0}^{r_{0}} \frac{d r}{e^{3 A} Z(\lambda)}}
$$


where $\theta_{U V}$ is the UV value for the QCD $\theta$-angle and the integer $k$ labels different large- $N_{c}$ vacua. In particular we reproduce the well known result that the $\theta$-dependent vacuum energy is to leading order in $1 / N_{c}$ proportional to $\operatorname{Min}_{k}\left(\theta_{U V}+2 \pi k\right)^{2}$ and relate the coefficient (topological susceptibility) to the standard QCD $\beta$-function as well as the axion $\beta$-function $Z(\lambda)$. One important corollary of our analysis is that $a(r)$, "the effective $\theta$-angle" vanishes as a power of the energy in the IR,

$$
\theta_{\text {eff }}(E) \sim E^{3}(\log E)^{\frac{1}{2}}
$$

This is an interesting result as it indicates that the YM dynamics screens the UV $\theta$-angle.

Before continuing we will comment on what we hope to achieve with this approach. As it will become evident, this phenomenological approach does not have at this point the status of well controlled approximation. Therefore at best we can hope to achieve the following:

1. To provide a successful and predictive phenomenological model with few parameters. A good analog of such a model is the Lund Monte Carlo that describes hadronization in high energy collisions using a basic string model and a dozen parameters.

2. If item 1 turns out to be successful, such a model can provide intuition and data, to guide serious searches for constructing a string theory for QCD.

3. It may provide hints for new phenomena in the theory. A good such example may turn out to be our observation that the IR $\theta$-angle in large- $N_{c}$ QCD vanishes.

This introduction summarizes the present paper as well its companion [20] that should be read as a natural continuation of this one. The structure of this paper is as follows.

In the next section we describe in detail the gravitational set up used to explore the duals of QCD-like gauge theories. In particular we analyze the general form of the scalar potential.

In section 3, we analyze the equations of motion for the coupled scalar-gravity system and derive the precise relation between the full $\beta$-function of the gauge theory and the scalar potential.

In section 4, we derive the UV asymptotics of the solutions close to the $A d S$ boundary, using the gauge theory input, namely asymptotic freedom. Then, we discuss the qualitative features of a class of IR asymptotics in the deep interior of the geometry that lead to confinement. This section also includes a detailed discussion of the $\alpha^{\prime}$ corrections near the boundary. In particular, we show that in the expansion 
near the UV, the $\alpha^{\prime}$ corrections are always accompanied with the scheme-dependent $\beta$-function coefficients.

Section 5 is devoted to specific examples of geometries that follow from a certain choice of the scalar potential. In section 5.1 we present a simple geometry that displays asymptotic freedom in the UV and linear confinement in the IR. This example is used in [20] to compute the glueball spectra. In section 5.2 we present a sample geometry that displays a Banks-Zaks type fixed point in the IR.

Section 6 investigates the fluctuations of the various fields in the geometry and how the dilaton potential (ie. the perturbative $\beta$-function) modifies the fluctuation equations near the UV boundary.

Various appendices detail our computations. In appendix A, we present a detailed analysis of the expansion near the UV. In appendix B we analyze a general form for the action of a dilaton-axion-gravity system that incorporates the $\alpha^{\prime}$ corrections by assuming general forms in the kinetic terms for these fields. We derive general solutions of the action and determine the $\alpha^{\prime}$ corrections (in the UV), to the various quantities used in the bulk-boundary identification. Appendix $\mathbb{Q}$ describes the geometry that follows from the naive effective potential which ignores the $\alpha^{\prime}$ corrections and studies the fluctuations around the AdS vacuum of this potential. In appendix D, we analyze the solutions to the coupled Einstein-scalar system with an exponential potential. We derive and classify all the solutions and describe the fixed points. Finally, appendix $\mathbb{E}$ presents another example of a geometry that approaches to a conformal fixed point in the IR with an exponential tail in the $\beta$-function.

As Part II of this work is an essential sequel of this paper we briefly review here its structure. It is mainly devoted to the analysis of the non-perturbative regime of our construction, i.e. to the IR properties of the 5D geometry. There, the general classification of IR asymptotics which lead to confinement is given. The qualitative features of the glueball spectra are discussed, and a relation is established between the existence of a mass gap and the confining property of the QCD string. The dependence of the spectrum on excitation number is discussed, and the IR asymptotics that lead to a linear glueball spectrum are identified. The analysis is extended to mesons, indicating that the setup provides a concrete realization of the holographic implementation of chiral dynamics proposed in [24]. The properties of the $5 \mathrm{~d}$ axion are discussed, and what they may imply for the QCD $\theta$-parameter. Finally, numerical computations of glueball spectra in concrete models are performed. The models are defined in terms of an exact $\beta$-function that interpolates between the desired UV and IR asymptotics. The detailed structure of the Part II can be found in its introduction. 


\section{Motivating the gravitational dual of a large- $\mathbf{N}_{c}$ gauge the- ory}

The gauge theories of interest in this paper, are four-dimensional $\mathrm{U}\left(\mathrm{N}_{c}\right)$ gauge theories at large $N_{c}$. In particular, we assume the presence of no further adjoint fields, and therefore, the holographic dual theory is expected to live in five dimensions. Fundamental matter can be present but we will assume here that the number of flavors $N_{f} \ll N_{c}$. Therefore, quarks can be eventually incorporated as four-brane probes inside the five-dimensional geometry.

\subsection{The spectrum}

The relevant non-critical string is therefore five-dimensional ${ }^{8}$. As there are no fermionic gauge-invariant operators in pure YM theory we do not expect the associated string theory to have space-time fermions, (No NSR and RNS sectors).

¿From the NSNS sector we will obtain as low lying fields a metric $g_{\mu \nu}$, a dilaton, $\phi$ and a two-index antisymmetric tensor $B_{\mu \nu}$. The generic bulk fields should be in oneto-one correspondence with the low-dimension gauge-invariant operators of the gauge theory. In particular the (classically) traceless stress tensor $T_{\mu \nu}=\operatorname{Tr}\left[F_{\mu \nu}^{2}-\frac{1}{4} \eta_{\mu \nu} F^{2}\right]$ is dual to the five-dimensional graviton $g_{\mu \nu} . \operatorname{Tr}\left[F^{2}\right]$ is dual to the dilaton $\phi . B_{\mu \nu}$ is expected to be dual, in analogy with $\mathcal{N}=4 \mathrm{sYM}$, to a higher dimension operator of the form $\operatorname{Tr}\left[d_{a b c} F^{a} F^{b} F^{c}\right]$ [3]. As it will be trivial in the vacuum solution, due to Lorentz invariance, we will neglect it in the sequel. It is however expected to generate a tower of $1^{-+}$glueballs in the theory.

The RR sector massless fields are summarized into the tensor product of two 5 -dimensional spinors. The product of two five-dimensional spinors gives the fields strengths $F_{0}, F_{1}, F_{2}, F_{3}, F_{4}, F_{5}$. There is an automatic duality condition for this product that relates $F_{5} \sim F_{0}, F_{4} \sim F_{1}$ and $F_{3} \sim F_{2}$. Therefore only $F_{0}, F_{1}, F_{2}$ are independent.

$F_{1}=d C_{0}$ generates an axion field $C_{0}=a$ that is dual to $\operatorname{Tr}[F \wedge F]$ in the YM theory. The dual of the axion field-strength is a four-form field strength $F_{4}$ and the associated three-form $C_{3}$ couples to domain walls (that separate different $k$-vacua).

$F_{5}=d C_{4} \sim F_{0}$ generates a four-form that seeds the $D_{3}$ branes responsible for the $U\left(N_{c}\right)$ gauge group. Its dual is a zero-form field strength that couples to bulk instantons.

Finally $F_{2}=d C_{1}$ generates a vector and its presence seems puzzling as there is no candidate YM operator that it is dual to. It turns out however that such a form couples minimally to the baryon density on flavor branes [24, and its presence is therefore justified. It is certainly trivial in the QCD vacuum as it will break fourdimensional Lorentz invariance otherwise. Its kinetic term is suppressed by $1 / N_{c}^{2}$ as

\footnotetext{
${ }^{8}$ For a similar discussion of the spectrum, see 25
} 
that of the axion. It can however be used to turn non-trivial baryon number densities in QCD. It is expected to generate a tower of spin-1 glueballs comparable in profile to the $0^{-+}$ones. We will analyze it further in [20].

We would like at this point to stress that the presence of RR fields is important with matching with the behavior expected from QCD. In particular, the special dependence on the dilaton of terms in the effective action containing RR fields (in particular the axion), is in complete agreement with the large $N_{c}$ suppression we expect for such terms in the gauge theory.

Flavor is expected to be generated by space filling $D_{4}+\bar{D}_{4}$ brane pairs filling (most of) the five dimensional bulk. They couple individually to a $C_{5}$ form. Overall neutrality is needed however as it equivalent to anomaly cancellation in the YM theory. ${ }^{9} \quad$ As the branes are space-filling, the coupling to $C_{5}$ will not play a role. Subleading couplings to other RR fields are essential though and will be discussed in 20.

The structure of the RR sector is similar to what we would obtain from an orientifold of the 0B theory [42, 43, 16] compactified to 5 dimensions. Several authors advocated the relevance of a closed string tachyon in the vacuum structure of QCD motivated by the ten dimensional type- 0 string theory. When $N_{f}=0$ we see no place for such a closed string tachyon, as there is no such low-dimension gauge-invariant operator in the gauge theory. If type-0 theory has any connection to pure YM theory, then the ten-dimensional tachyon must become rather massive in the fivedimensional theory. Of course if we add $N_{f}$ flavor branes with $N_{f} \ll N_{c}$ then the open string tachyon is essential for understanding meson physics, [24]. However, the tachyon profile does not back-react to correct the gauge theory vacuum in this case. A (closed) string tachyon may be relevant in understanding the vacuum structure of QCD in the Veneziano limit, $N_{f} \sim N_{c}$. In this case it is the avatar of the open string tachyon of the flavor branes, which, because of their large number is becoming effectively a closed string state (in the sense that it affects the vacuum structure at leading order in $1 / N_{c}$ ).

To summarize, when $N_{f}=0$ the relevant propagating bulk fields are $g_{\mu \nu}, \phi, a$, while there is also a non-propagating $F_{5}$ field strength. When $N_{c} \gg N_{f} \neq 0$, there is also an open string tachyon $T$ (together with gauge fields on the flavor branes), which is a $N_{f} \times N_{f}$ complex matrix charged under gauge fields of the $U\left(N_{f}\right)_{L} \times U\left(N_{f}\right)_{R}$ symmetry, 24]. In this paper we will not consider the dynamics of flavor. But we will include the contribution of the tension of flavor branes in the effective action in this section only to indicate the scaling of various terms in the effective dilaton potential.

\footnotetext{
${ }^{9}$ This appears to be rather general in holographic setups. Cancellation of bulk RR charges is equivalent to the absence of gauge anomalies of the large- $N_{c}$ boundary theory.
} 


\subsection{The associated branes}

There are several potential branes associated to the various bulk form fields that exist in the theory. The RR four-form couples to $\mathrm{D}_{3}$ branes, responsible for generating the gauge group.

The RR zero form, dual to the YM $\theta$-angle couples electrically to $D_{-1}$ instantons, which should be indeed the YM instantons. It is interesting to mention here that such bulk instanton solutions in the $A d S_{5}$ case were identified with the standard YM instanton solutions with the dependence on the radial holographic coordinate playing the role of instanton size. In our case we expect that small instantons, as they will be close to the $\mathrm{AdS}_{5}$ boundary, resemble almost accurately the standard YM Instantons. Large instantons however, feel the non-trivial IR geometry and their form is expected to part substantially from the standard YM form. It may be interesting to study them as they might indicate the correct instanton measure in the IR of YM theory. This should be the five-dimensional volume form in the non-trivial metric that describes the YM vacuum, together with a power of the dilaton that needs to be determined.

The magnetic duals of the $D_{-1}$ instantons are $D_{2}$ membranes and they couple minimally to the dual $C_{3}$ form. They are domain walls in four-dimensions separating different $k$-vacua. A point-like $D_{2}$ generates a monodromy for the bulk axion field in the transverse two dimensional space.

There are also point-like $D_{0}$ branes coupled to the RR one-form $C_{1}$. They are the baryon vertices which can tie up $N_{c}$ quarks to form a large- $N_{c}$ baryon. Their duals are $D_{1}$ strings that couple to the dual two-form $C_{2}$, which represent the flux tubes stretched among magnetic quark sources. They will be used in 20] to investigate the interaction between magnetic quarks in confining vacua.

Finally in the NSNS sector we have $\mathrm{NS}_{0}$ branes that are five-dimensional duals of the fundamentals strings. They are charged magnetically under the NSNS twoform $B_{\mu \nu}$, and couple directly to the flavor $\mathrm{U}(1)$ gauge-fields. The S-duality map would suggest that they can be viewed as vertices for magnetic baryons. This is in agreement with the fact that such magnetic baryons are expected to have masses that scale as $\mathcal{O}\left(N_{c}^{2}\right)$ compared to ordinary baryons whose masses are of order $\mathcal{O}\left(N_{c}\right)$. The reason is that they contain $N_{c}$ magnetic quarks, each having a mass of order $N_{c} \cdot{ }^{10}$

\subsection{The effective action}

The (minimal) two-derivative effective action of the perturbative string theory is

$$
S_{5}=M^{3} \int d^{5} x \sqrt{g}\left[e^{-2 \phi}\left(R+4(\partial \phi)^{2}+\frac{\delta c}{\ell_{s}^{2}}\right)-\frac{1}{2 \cdot 5 !} F_{5}^{2}-\frac{1}{2} F_{1}^{2}-\frac{N_{f}}{\ell_{s}^{2}} e^{-\phi}\right]
$$

\footnotetext{
${ }^{10}$ See however a slightly different interpretation in $[25$.
} 
where as usual $F_{1}=\partial_{\mu} a$ and we have used the 5 -form instead of the zero form. The last term is due to space filling $D_{4}-\bar{D}_{4}$ brane pairs, with $N_{f}$ is proportional to the number of flavor branes. We have explicitly indicated the string scale $\ell_{s}$ and the Planck scale

$$
M^{3}=\frac{1}{g_{s}^{2} \ell_{s}^{3}} \quad, \quad \delta c=10-D=5
$$

Anticipating the connection with the gauge theory let us define,

$$
\lambda=N_{c} e^{\phi}
$$

Passing to the Einstein frame by $g_{\mu \nu}=\lambda^{\frac{4}{3}} g_{\mu \nu}^{E}$ we obtain:

$S_{5}=M^{3} \int d^{5} x \sqrt{g}\left[N_{c}^{2}\left(R-\frac{4}{3} \frac{(\partial \lambda)^{2}}{\lambda^{2}}+\frac{\delta c}{\ell_{s}^{2}} \lambda^{\frac{4}{3}}\right)-\frac{\lambda^{-\frac{10}{3}}}{2 \cdot 5 !} F_{5}^{2}-\frac{\lambda^{2}}{2}(\partial a)^{2}-N_{c}^{2} \frac{N_{f}}{N_{c} \ell_{s}^{2}} \lambda^{\frac{7}{3}}\right]$.

We are now at the point where we can solve for the five-form (that controls the number of $D_{3}$ branes). Starting from the Einstein frame action

$$
\delta S_{5}=-\frac{M^{3}}{2 \cdot 5 !} \int d^{5} x \sqrt{g} \lambda^{-\frac{10}{3}} F_{5}^{2}
$$

the equations of motion are $d^{*}\left(\lambda^{-\frac{10}{3}} F_{5}\right)=0$ with the solution

$$
F_{\mu_{1} \cdots \mu_{5}}=\frac{N_{c}}{\ell_{s}} \lambda^{\frac{10}{3}} E_{\mu_{1} \cdots \mu_{5}} \equiv \frac{N_{c}}{\ell_{s}} \lambda^{\frac{10}{3}} \frac{\epsilon_{\mu_{1} \cdots \mu_{5}}}{\sqrt{-\operatorname{det} g}}
$$

where $\mathrm{E}$ is the totally antisymmetric tensor and $N_{c}$ is dimensionless and proportional to the number of colors. This is the definition of $N_{c}$, and it is obviously unormalized, unless we know explicitly the $D_{3}$ brane solution of the associated string theory. Inserting back in the equations of motion and from this reconstructing the action, we obtain

$$
\delta \tilde{S}_{5}=-\frac{M^{3} N_{c}^{2}}{2} \int d^{5} x \sqrt{g} e^{\frac{10}{3} \phi}
$$

so that

$$
S_{5}=M^{3} N_{c}^{2} \int d^{5} x \sqrt{g}\left[R-\frac{4}{3} \frac{(\partial \lambda)^{2}}{\lambda^{2}}-\frac{\lambda^{2}}{2 N_{c}^{2}}(\partial a)^{2}+V(\lambda)\right]
$$

with

$$
V(\lambda)=\frac{\lambda^{\frac{4}{3}}}{\ell_{s}^{2}}\left[\delta c-x \lambda-\frac{1}{2} \lambda^{2}\right]
$$

Here we defined the ratio of number of flavors over the number of colors,

$$
x=\frac{N_{f}}{N_{c}} .
$$


This potential is analyzed in detail in appendix C. It has a minimum at a finite $\mathcal{O}(1)$ value of the $t$ 'Hooft coupling ${ }^{11}$ 2.3.

This gives rise to an $A d S_{5}$ solution that was found and analyzed in [8], where it was conjectured to describe the Banks-Zaks fixed points (for $N_{f} \sim N_{c}$, i.e. $x \sim$ 1). One might think that a dilaton flow around the $A d S_{5}$ solution might describe the gauge theory physics we are seeking. A study of the dilaton perturbations in appendix $\square$ shows however that when $N_{f} \ll N_{c}$, the dimension of the operator dual to the dilaton perturbation is well above 6 , and this does not compare well with the dimension-4 perturbation we are looking for. On the other hand, it is expected that for $N_{f} \sim N_{c}$, the solution will be importantly affected by the tachyon field. Therefore an analysis in this case that neglects the tachyon effects may be unreliable. Thus it is plausible that the $A d S_{5}$ solution found is relevant for gauge theories in their Veneziano limit, as argued in [B]

Another problem of this AdS vacuum is that, as we show in appendix $\mathrm{Q}$, the $\mathrm{t}$ 'Hooft coupling at that point is of order one, and this is not something we expect in the UV region of QCD.

We are therefore led to the suggestion that the UV of QCD should be described by the $\phi \rightarrow-\infty$ asymptotics of the potential in (2.8). To test this claim, we must study the asymptotic solution of the classical equations and compare it to expectations from QCD. To do this we make a Poincaré-invariant ansatz for the metric. The equations of motion in the covariant form are,

$$
E_{\mu \nu}-\frac{4}{3}\left[\partial_{\mu} \phi \partial_{\nu} \phi-\frac{1}{2}(\partial \phi)^{2} g_{\mu \nu}\right]-\frac{1}{2} g_{\mu \nu} V=0 \quad, \quad \square \phi+\frac{3}{8} \frac{d V(\phi)}{d \phi}=0 .
$$

with the Einstein tensor defined as,

$$
E_{\mu \nu}=R_{\mu \nu}-\frac{1}{2} R g_{\mu \nu},
$$

We neglect the QCD axion $a$ in our system as it is not expected to affect this solution. ${ }^{12}$

Let us digress here to introduce the following two coordinate systems we use in the paper. The "conformal" coordinate system is given by,

$$
d s^{2}=e^{2 A(r)}\left[d r^{2}+\eta_{i j} d x^{i} d x^{j}\right],
$$

\footnotetext{
${ }^{11}$ We stress again that this is the definition we use for the 't Hooft coupling and it is unormalized.

${ }^{12}$ In large- $N_{c}$ theories with strong IR dynamics like QCD, Witten has argued long time ago 26, that although instanton effects seem negligible at large- $N_{c}$, there is non-trivial $\theta$ dependence in QCD dynamics. This is in particular responsible for the resolution of the $\eta^{\prime}$ mass problem via the Witten-Veneziano mechanism, [27]. Although we expect via such considerations $a$ to have a nontrivial profile in five dimensions, its "order of magnitude" is $\mathcal{O}\left(1 / N_{c}\right)$ and therefore its back-reaction to the other bulk fields can be neglected to leading order [28].
} 
and the "domain wall" coordinate system is given by,

$$
d s^{2}=e^{2 A(u)}\left[\eta_{i j} d x^{i} d x^{j}\right]+d u^{2} .
$$

The two are related by ${ }^{13}$,

$$
d u=e^{A(r)} d r
$$

The advantage of the conformal variable $r$ is that in an asymptotically AdS background, it is directly related to the energy of the gauge theory as $E=1 / r$ in the far UV. Also the corrections to the AdS metric that we describe in this paper take the simple form of $\log r$. On the other hand, the domain-wall coordinate $u$ facilitates the solution to the differential equations and uncovers the relation between the integration constants and the related gauge theory quantities. Henceforth, we will denote by prime, a derivative with respect to $u$ and by dot, a derivative with respect to $r$.

The equations of motion in the conformal coordinate read

$$
\begin{gathered}
12 \dot{A}^{2}-\frac{4}{3} \dot{\phi}^{2}-e^{2 A} V=0 \quad, \quad 6 \ddot{A}+6 \dot{A}^{2}+\frac{4}{3} \dot{\phi}^{2}-e^{2 A} V=0 \\
\ddot{\phi}+3 \dot{A} \dot{\phi}+\frac{3}{8} e^{2 A} \frac{d V}{d \Phi}=0
\end{gathered}
$$

We shall also need the Einstein's equations and the scalar field equation in the domain-wall coordinate, in the following form:

$$
\begin{gathered}
A^{\prime 2}=\frac{\left(\phi^{\prime}\right)^{2}}{9}+\frac{V}{12} \quad, \quad A^{\prime \prime}=-\frac{4}{9}\left(\phi^{\prime}\right)^{2} \\
\phi^{\prime \prime}=-4 A^{\prime} \phi^{\prime}-\frac{3}{8} \frac{d V(\phi)}{d \phi} .
\end{gathered}
$$

What type of solution do we expect in the UV regime $(r \rightarrow 0)($ or $u \rightarrow-\infty)$ ? As QCD becomes scale invariant at $E=\infty$, we expect the space to asymptote to $A d S_{5}$. Moreover as $\frac{1}{\lambda} \sim \log E$ we expect that $e^{-\phi} \sim-\log r$. Therefore, the geometry is expected to be $\mathrm{AdS}_{5}$ modulo logarithmic corrections. This asymptotic expansion is described in detail in appendix $\mathrm{A}$.

The potential in (2.8) as $\phi \rightarrow-\infty$, behaves as $V \sim e^{a \phi}$ with $a=\frac{4}{3}$. In appendix D we analyze in detail the solutions to the single exponential potential. We show that in such cases the asymptotics of the metric is quite different from the expected behavior outlined above. Indeed one finds, as $r \rightarrow 0$,

$$
e^{A(r)} \sim \begin{cases}r^{\frac{16}{9 a^{2}-16}} & \text { if } a \neq \frac{4}{3} \\ e^{r} & \text { if } a=\frac{4}{3}\end{cases}
$$

\footnotetext{
${ }^{13}$ For simplicity we denote the warp factor in both of the coordinate systems by $A$ i.e. $A(u)=$ $A(r(u))$.
} 
In fact, the dilaton potential that has the desired behavior in the UV, must asymptote to a (positive) constant, as $\phi \rightarrow-\infty$. The natural question is whether such a constant can appear from string theory considerations.

In five dimensions there are higher-order $\alpha^{\prime}$ corrections, that contribute to the dilaton potential. This may sound implausible, as such corrections carry a number of derivatives and are not therefore part of the potential. However, it should be remembered that the bulk theory contains the (non-propagating) four-form $C_{4}$, which is responsible for the charge of $D_{3}$ branes sourcing the bulk solution. Its field strength is $F_{5}$. At the quadratic level, it generates the $e^{\frac{10}{3} \phi}$ term in the potential, upon dualization. It goes without saying that its higher-derivative avatars in the effective action will contribute several more terms in the dilaton potential. For example the n-th order term (on the sphere) in the $\alpha^{\prime}$ expansion is

$$
S_{n} \sim \frac{M^{3}}{\ell_{s}^{2-2 n}} \int d^{5} x e^{2(n-1) \phi} \sqrt{g}\left(F_{5}\right)^{2 n}
$$

where the dilaton dependence is dictated by the special transformation properties of RR field strengths. As explained in detail in appendix B, upon dualization, such terms translate into a dilaton potential of the form

$$
V_{F_{5}} \sim \frac{e^{\frac{4}{3} \phi}}{\ell_{s}^{2}} \sum_{n=0}^{\infty} a_{n}\left(N_{c} e^{\phi}\right)^{2 n}
$$

where the $a_{n}$ are dimensionless numbers reflecting the coefficients of the higher derivative terms like (2.20). Note that we included the central charge deficit as the first term in this potential.

This potential is remarkable for two reasons. Firstly, it is a function of the 't Hooft coupling $\lambda \sim N_{c} e^{\phi}$, and remains finite in large- $N_{c}$ perturbation theory. The second is that it has a regular expansion for small 't Hooft coupling. On the other hand it does not contain the term we are looking for, namely a (positive) constant. In appendix B.3 we indicate how such an effective term, generating an asymptotic AdS regime can be generated by higher curvature corrections. We will not however pursue this line of thought here, instead we will add such a term to the dilaton potential by fiat, as was also done in [12] for example.

The next question to address is what the other terms in the potential should be in order to reproduce the expected logarithmic running of the gauge theory coupling constant $\lambda$, that is compatible with QCD perturbation theory. To answer this question, one must identify the UV energy variable, and this is essentially the coordinate $r$ up to logarithmic corrections. Matching then the expected equation

$$
\frac{d \lambda}{d \log r}=\beta(\lambda)=-b_{0} \lambda^{2}+b_{1} \lambda^{3}+b_{2} \lambda^{4}+\cdots
$$


with the bulk equations of motion in (2.16), (2.17) we obtain that the weak-coupling expansion of the potential should be

$$
V(\lambda)=\sum_{n=0}^{\infty} V_{n} \lambda^{n}
$$

To leading order, $\lambda \sim N_{c} e^{\phi}$ as can be ascertained by the world-volume couplings of $D_{3}$ branes $^{14}$. Higher $\alpha^{\prime}$ corrections involving the five-form on $D_{3}$ branes can change this identification to

$$
\lambda=\sum_{n=0}^{\infty} c_{n}\left(N_{c} e^{\phi}\right)^{(2 n+1)}
$$

as is shown in appendix B.2. In case of $\mathcal{N}=4 \mathrm{YM}$, the leading coefficient is $c_{0}=4 \pi$. However, in general we consider a possible multiplicative renormalization in the identification of $\lambda$, hence we keep $c_{0}$ arbitrary. Moreover, changing $c_{0}$ is equivalent to changing $b_{0}$ the first $\beta$-function coefficient. Therefore we will eventually set $c_{0}=1$ and we will allow ourselves to vary $b_{0}$ to reflect this normalization ambiguity.

Such corrections affect the $\beta$-function beyond the first two non-trivial orders, (associated to $\left.b_{0}, b_{1}\right)$. Since higher coefficients are non-universal, we choose to neglect the renormalizations in (2.24), and set $c_{n}=0$ for $n \geq 1$. In the analysis of appendix A we have set $c_{0}$ to 1 . Reinstating it here we obtain

$$
\begin{aligned}
& V(\lambda)=\frac{12}{\ell^{2}}\left[1+\frac{8}{9}\left(b_{0} \lambda\right)+\frac{23-36 \frac{b_{1}}{b_{0}^{2}}}{3^{4}}\left(b_{0} \lambda\right)^{2}-2 \frac{324 \frac{b_{2}}{b_{0}^{3}}+124+189 \frac{b_{1}}{b_{0}^{2}}}{3^{7}}\left(b_{0} \lambda\right)^{3}+\mathcal{O}\left(\lambda^{4}\right)\right] \\
& =\frac{12}{\ell^{2}}\left[1+\frac{8}{9}\left(b_{0} c_{0} N_{c} e^{\phi}\right)+\frac{23-36 \frac{b_{1}}{b_{0}^{2}}}{3^{4}}\left(b_{0} c_{0} N_{c} e^{\phi}\right)^{2}-2 \frac{324 \frac{b_{2}}{b_{0}^{3}}+124+189 \frac{b_{1}}{b_{0}^{2}}}{3^{7}}\left(b_{0} c_{0} N_{c} e^{\phi}\right)^{3}+\cdots\right]
\end{aligned}
$$

where $\ell$ is the AdS radius. We conclude that without knowledge of $c_{0}$, the effective one-loop term $b_{0}^{e f f}=b_{0} c_{0}$ is unknown.

In appendix $\mathrm{A}$ it is shown that changing the constant $b_{0}$ amounts to a redefinition of the perturbative QCD scale $\Lambda$ as well as a redefinition of the non-universal coefficients of the $\beta$-function. This is nicely reflected in the fact that the dilaton potential (2.25) depends only on the invariant ratios $b_{i} / b_{0}^{i+1}$. Moreover as shown in the same appendix, the first logarithmic correction to the UV AdS metric is universal, and in particular insensitive to either $b_{0}$, or $c_{0}$. In view of the above, we will postulate from now on, a dilaton potential with a weak coupling expansion as in (2.25) with $c_{0}=1$.

\footnotetext{
${ }^{14}$ If the space has more than five dimensions or there are other bulk scalar field involved, this identification can change. However this is not the case here. Another issue involves finite renormalizations of the 't Hooft coupling identification. Such corrections cannot be excluded but they cannot affect the qualitative analysis, as they involve a constant rescaling of the dilaton. We choose therefore to ignore them.
} 


\section{Holographic large $\mathbf{N}_{c}$ YM: the vacuum structure}

Before we embark on a study of the solution to the equations of motion that describes the vacuum structure of the theory, we review the action we are going to use

$$
S=M^{3} N_{c}^{2} \int d^{5} x \sqrt{g}\left[R-\frac{4}{3} \frac{(\partial \lambda)^{2}}{\lambda^{2}}-\frac{Z(\lambda)}{2 N_{c}^{2}}(\partial a)^{2}+V(\lambda)\right]
$$

with

$$
V(\lambda)=\frac{12}{\ell^{2}}\left(1+\sum_{n=1}^{\infty} V_{n} \lambda^{n}\right)=\frac{12}{\ell^{2}}\left(1+\frac{8}{9} b_{0} \lambda+\frac{23 b_{0}^{2}-36 b_{1}}{81} \lambda^{2}+\cdots\right)
$$

and where we have added a general kinetic function $Z(\lambda)$ in front of the axion. As the axion kinetic energy is suppressed by $1 / N_{c}^{2}$ it will be neglected in the following investigation of the vacuum solution. In the action (3.1), $N_{c}$ appears only as an overall coefficient, i.e. it only changes the definition of the 5-dimensional Planck scale. Therefore in the large $N_{c}$ limit, quantum corrections can be safely neglected, as in the standard, critical $A d S / C F T$ correspondence. All kinetic and potential terms are independent of $N_{c}$ when written in terms of $\lambda$. Therefore, it is useful to define an "effective" dilaton field by

$$
\Phi \equiv \log \lambda=\phi+\log N_{c}
$$

From now on, this is the field we will use, and we will refer to it as the "dilaton" without any further specification.

\subsection{General geometry and the geometric invariants}

In this section we introduce a rewriting of the equations of motion that facilitates the construction of the desired 5D geometry and its connection to the gauge theory. We allow for a general dilaton potential $V(\Phi)$ in (3.1) that admits an expansion of the form (3.2) for small $\lambda$.

We start from the domain-wall parametrization of the metric in (2.14). We use the Einstein's equations (2.18) and the scalar field equation (2.19). It is a wellknown fact that these second-order equations can be reduced to a system of firstorder equations ${ }^{15}$. This is generally done by introducing a superpotential ${ }^{16}$. Here we find it more convenient to rewrite the equations in terms of the derivative of the superpotential with respect to the dilaton. For this purpose, we introduce the phase space variable $X$ :

$$
X=\frac{\Phi^{\prime}}{3 A^{\prime}}
$$

\footnotetext{
${ }^{15}$ This well-known technique in differential equations was discussed in this context in [29.

${ }^{16}$ It goes without saying that this amounts to choosing one of the two initial conditions for the dilaton.
} 
This variable is useful in our set-up as it is closely related to the gauge theory $\beta$ function.

In terms of $X$, the second order system can be rewritten as the following firstorder system of equations,

$$
\begin{aligned}
\left(\Phi^{\prime}\right)^{2} & =\frac{3}{4} V_{0} X^{2} e^{-\frac{8}{3} \int_{-\infty}^{\Phi} X d \Phi}, \\
\left(A^{\prime}\right)^{2} & =\frac{V_{0}}{12} e^{-\frac{8}{3} \int_{-\infty}^{\Phi} X d \Phi}, \\
\frac{d X}{d \Phi} & =\left(8 X+3 \frac{d \log V}{d \Phi}\right) \frac{\left(X^{2}-1\right)}{6 X} .
\end{aligned}
$$

Here, $V_{0}>0$ is the asymptotic value of the dilaton potential as $\Phi \rightarrow-\infty$. Given $X$ as a function of $\Phi$, one solves (3.5) to determine the dilaton and (3.6) to obtain the geometry. Our strategy in solving the system of equations is essentially reversing (3.7) and determining the potential $V(\Phi)$, given the function $X(\Phi)$. In the next subsection, we explain how to determine $X$, by using the gauge theory input. It is completely determined by the full $\beta$-function of the gauge theory. We analyze certain fixed points of (3.7) in the appendix $\mathrm{D}$.

Here, we assume that $X$ is known, and calculate the invariants of the geometry. This is necessary in order to understand the singularity structure of the solution in the IR.

We solve (3.7) for $V$ as follows:

$$
V(\Phi)=V_{0}\left(1-X^{2}\right) e^{-\frac{8}{3} \int_{-\infty}^{\Phi} X d \Phi},
$$

where $V_{0}>0$.

The leading scalar invariants, that are constructed from the Einstein tensor (defined in (2.12)) are,

$$
\begin{aligned}
E & \equiv E_{\mu}{ }^{\mu}=30\left(A^{\prime}\right)^{2}+12 A^{\prime \prime}, \\
E_{\mu \nu} E^{\mu \nu} & =36\left(5\left(A^{\prime}\right)^{4}+4 A^{\prime \prime}\left(A^{\prime}\right)^{2}+\left(A^{\prime \prime}\right)^{2}\right) .
\end{aligned}
$$

Using the equation of motion (2.18) together with (3.5) and (3.6), we obtain the following results for the first-order invariant:

$$
E=V_{0}\left(\frac{5}{2}-4 X^{2}\right) e^{-\frac{8}{3} \int_{-\infty}^{\Phi} X d \Phi},
$$

We show in the next section that the presence of an asymptotic AdS geometry near the boundary requires $X \rightarrow 0$ there. From (3.11) we observe that $E \rightarrow 5 V_{0} / 2$ there, as expected from the AdS geometry. This provides a consistency check of our formulae. The second order invariant is,

$$
E \cdot E=\frac{V_{0}^{2}}{8}\left(2-11 X^{2}\left(1-X^{2}\right)\right) e^{-\frac{16}{3} \int_{-\infty}^{\Phi} X d \Phi} .
$$


We also present the relation of the phase variable and the superpotential. In 5D, the superpotential is obtained from the scalar potential by solving,

$$
W^{2}-\left(\frac{3}{4}\right)^{2}\left(\frac{\partial W}{\partial \Phi}\right)^{2}=\left(\frac{3}{4}\right)^{3} V(\Phi)
$$

The first order equations that follow from this superpotential are,

$$
A^{\prime}=-\frac{4}{9} W \quad, \quad \Phi^{\prime}=\frac{d W}{d \Phi} .
$$

Comparison of (3.14) with (3.6) and (3.5) shows that the superpotential is given as,

$$
W=\frac{9 \sqrt{V_{0}}}{8 \sqrt{3}} e^{-\frac{4}{3} \int_{-\infty}^{\Phi} X d \Phi} .
$$

It is also useful to note that $X$ is proportional to the derivative of the logarithm of the superpotential:

$$
X=-\frac{3}{4} \frac{d \log W}{d \Phi}
$$

\subsection{The phase variable $X$ and the $\beta$-function}

In this section, we show that given i) the relation between the 't Hooft coupling $\lambda$ and $\phi$ and ii) the relation between energy scale of the gauge theory, and the radial variable of the $5 \mathrm{D}$ geometry, one can relate the phase space variable that we introduced in (3.4) to the $\beta$-function of the gauge theory. Both relations receive $\alpha^{\prime}$ corrections, but we shall first ignore them and consider their effects in section 4.2 .

To fix the first relation, we consider the metric (2.14) in the Einstein frame. There are at least two arguments that lead to the energy-radius dependence: The first is the Polchinski-Strassler type of argument [31] that uses the gravitational red-shift to relate

$$
E=\sqrt{g_{00}} E_{b u l k}
$$

where the LHS is the energy in $4 \mathrm{D}$ field theory measured by an observer on the boundary of the 5D geometry and the RHS is the energy of a point-like excitation of the gravitational system. This gives,

$$
\log (E) \propto A
$$

The second argument is to make an infinitesimal shift $u \rightarrow u+\delta$ in the metric and to observe that this shift can be produced by a dilatation $x^{\mu} \rightarrow(1+\rho) x^{\mu}$. This dilatation changes the $4 \mathrm{D}$ energy by, $\log (E) \rightarrow \log (E)-\rho$. Equivalently, one makes the above shifts in $u$ and $x$ and demands that the metric does not change to first order in $\rho$. This fixes,

$$
\rho=-A^{\prime} \delta
$$


hence one arrives at the same result (3.18).

We note that the relation (3.18) is the most natural in an asymptotically AdS geometry where $A \rightarrow-u / l$ as $u \rightarrow-\infty$ and one identifies $\log E=u$ in the original AdS/CFT correspondence ([33). However, we stress that any function whose leading behavior agrees with (3.18) in the UV is a candidate definition for the field theory energy.

Another necessary condition is to have a monotonically decreasing function of the radial variable $u$. This is necessary in order to have a well-defined direction for the renormalization group flow. From the Einstein's equation (2.18), it is clear that (3.18) is guaranteed to satisfy this criterion. This is because, it follows from (2.18) that $A^{\prime}$ is a monotonically decreasing function of $u$. Since it is already negative at $u=-\infty, A^{\prime}$ is forced to be negative in the entire range of $u$. Thus, $A$ itself should be a monotonically decreasing function of $u$.

We should also note that the scale factor of the metric in the Einstein frame is expected to be associated to the C-function of the associated theory. In particular with our definition we expect that $E^{4} \sim e^{4 A}$ is proportional to the number of degrees of freedom of the theory with energy up to $E$.

Now, we come to the identification of the $\beta$-function. As discussed in Section 2 , we identify the 't Hooft coupling as:

$$
\lambda=N_{c} e^{\phi} \equiv e^{\Phi} .
$$

where we chose to set the coefficient $c_{0}$ to one here by reabsorbing it in the potential.

On the other hand, the $\beta$-function of the gauge theory is given by,

$$
\beta(\lambda)=\frac{d \lambda}{d \log (E)}=\frac{d \lambda}{d A} .
$$

The definition of the phase variable $X$ in (3.4) together with the gauge theorygeometry relations (3.18) and (3.19) leads to the following identification of the bulk and boundary quantities:

$$
X=\frac{\beta(\lambda)}{3 \lambda} .
$$

In the next section, we shall make use of (3.21) in order to formulate restrictions on the geometry that follow from the desired properties of the gauge theory.

\section{Asymptotics of the geometry}

\subsection{Asymptotic freedom and the UV geometry}

In an asymptotically free gauge theory, the 't Hooft coupling behaves as,

$$
\lambda \propto \frac{1}{\log E}, \quad \text { for large } E .
$$


Using our identification of the energy scale of the gauge theory with the scale factor of the metric in the Einstein frame, i.e. $\log E \propto A$, we learn that this requirement means, $\Phi \propto-\log A$, or in terms of the phase-space function $X$,

$$
X \propto-\frac{1}{A} \propto-\lambda .
$$

Making use of (4.2) in (3.8), one learns that the geometry dual to an asymptotically free gauge theory, requires the following form of the scalar potential near $\lambda=0$ :

$$
V=V_{0}+V_{1} \lambda+\cdots
$$

as was already announced in section 2. Therefore asymptotic freedom in the UV requires an asymptotically $\mathrm{AdS}_{5}$ geometry. In addition to this, it requires an expansion of the form (4.3) around $\lambda=0$. In particular, the presence of the first order term $V_{1}$ is crucial for the (leading) logarithmic running of the coupling constant.

One can easily make a consistency check on the condition, (4.2). Having an AdS geometry in the UV gives $A \propto u$ near the boundary $(u \rightarrow \infty)$. Therefore, near the boundary we have,

$$
\lambda \propto \frac{1}{u} .
$$

Using the eq. (3.6) or (3.5) we see that this results in the following asymptotic form for $X$ :

$$
X \propto \lambda+\mathcal{O}\left(\lambda^{2}\right), \quad \text { as } \lambda \rightarrow 0 .
$$

In particular, as discussed in section 2, the simple set up in (2.4) does not satisfy this asymptotics. Although we presented ideas on how the two requirements can be reconciled, in this work we will assume the presence of the suitable dilaton potential.

\subsection{The $\alpha^{\prime}$ corrections}

We now generalize the above discussion by taking into account the possible $\alpha^{\prime}$ corrections near the boundary. As is clear from (3.8) the requirement (4.3) follows from requiring (4.5). We would like to determine the asymptotics of $X$ by taking into account the possible corrections that fall into the following three classes. First, there is a subclass of the derivative corrections to the Einstein-Hilbert action studied in appendix $(\mathrm{B})$. Second, there are the derivative corrections to the probe $\mathrm{D}_{3}$ brane action studied in appendix (B.2). The combined effect of these corrections can be stated as,

$$
\lambda=\lambda_{s}\left(1+c_{1} \lambda_{s}^{2}+\left(2 a_{2} c_{1}+\frac{c_{2}}{2}+c_{1}^{2}\right) \lambda_{s}^{4}+\mathcal{O}\left(\lambda_{s}^{6}\right)\right)
$$

Here,

$$
\lambda_{s}=e^{\phi} N_{c},
$$

is a purely bulk quantity, while we use $\lambda$ to denote the 't Hooft coupling of the gauge theory. The unknown coefficients $a_{i}$ and $c_{i}$ are defined in (B.2) and (B.40). 
The third type of correction involves the definition of the gauge theory energy scale in terms of the metric scale factor. Using the fact that all of the functions in the geometry can be expressed in the variable $\lambda_{s}$, and that, in the far UV, the radial variable in AdS exactly corresponds to the energy scale, we may write,

$$
\frac{d \log E}{d A}=f\left(\lambda_{s}\right)=1+f_{1} \lambda_{s}^{2}+f_{2} \lambda_{s}^{4}+\cdots
$$

with $f_{i}>0$, for all $i$. This expansion anticipates that the variable $\lambda_{s}$ asymptotes to zero in the UV, and this will be checked below. We note that a specific choice for the function $f\left(\lambda_{s}\right)$ corresponds to a specific scheme ${ }^{17}$ in computing the $\beta$-function of the field theory. The reason that the expansion variable is $\lambda_{s}^{2}$ instead of $\lambda_{s}$ is due to the form of the $\alpha^{\prime}$-expansion, as derived in Appendix B.3.

Now, we can connect $X$ to $\beta$ :

$$
\beta=\frac{d \lambda}{d \log E}=\frac{d \lambda}{d \lambda_{s}} \frac{d \lambda_{s}}{d A} \frac{d A}{d \log E}=\frac{d \lambda}{d \lambda_{s}} 3 X \lambda_{s} f\left(\lambda_{s}\right)^{-1} .
$$

Eqs. (4.6) and (4.8) give,

$$
\begin{aligned}
X= & -\frac{b_{0}}{3} \lambda+\frac{b_{1}}{3} \lambda^{2}+\left(\frac{b_{2}}{3}-\frac{4}{3} c_{1} b_{0}+\frac{f_{1} b_{0}}{3}\right) \lambda^{3}+\left(\frac{b_{3}}{3}+\frac{4}{3} c_{1} b_{1}-\frac{f_{1} b_{1}}{3}\right) \lambda^{4} \\
& +\frac{1}{3}\left(b_{4}+b_{2}\left(4 c_{1}-f_{1}\right)+2 c_{1} f_{1} b_{0}+c_{1} b_{0}^{2}-f_{1}^{2} b_{0}+f_{2} b_{0}-4 a_{2} c_{1} b_{0}-2 c_{2} b_{0}\right) \lambda^{5}+\cdots
\end{aligned}
$$

We remark that the first two terms are completely determined by the scheme independent gauge theory input, i.e. the first two $\beta$-function coefficients, $b_{0}$ and $b_{1}{ }^{18}$. A very interesting corollary of (4.10) is that the unknown coefficients of the $\alpha^{\prime}$ expansion, namely $a_{i}, c_{i}$ and $f_{i}$ are always accompanied by the scheme dependent $\beta$-function coefficients $b_{2}, b_{3}, \ldots$. This, for example, makes it possible to set all the $f_{i}$ to zero by choosing an appropriate scheme in the computation of the full $\beta$-function. In what follows we shall assume that this scheme is chosen and we shall set $f_{i}=0$ for all $i$. We call it the "holographic scheme". A final observation in (4.10) is that the $\alpha^{\prime}$ corrections to the effective action discussed in Appendix B, (associated to $a_{i}$ ), enter first at the fourth order in the expansion.

So far we have dealt with the $\alpha^{\prime}$ corrections near the boundary. The possible corrections in the IR regime shall be discussed in section 4.4.

\subsection{The UV geometry}

We can obtain the UV asymptotics of the metric by using (4.10) and the formalism of the previous subsection. The asymptotic form of the metric to leading, subleading

\footnotetext{
${ }^{17}$ The scheme dependence of the renormalization group running in the holographic setup has been discussed in [32].

${ }^{18}$ As explained earlier, in the correspondence of the bulk and the boundary quantities, neither $b_{0}$ nor $b_{1}$ but only the ratio $b_{1} / b_{0}^{2}$ has an invariant meaning as we do not know the correct normalization of $\lambda$
} 
and next to subleading order, turns out to be independent of the corrections discussed above:

$$
d s^{2}=e^{2 \frac{u}{\ell}} u^{\frac{8}{9}+2 b\left(\frac{4}{9}-b\right) / u+\cdots} d x^{2}+d u^{2},
$$

where $\ell$ is the radius of $\mathrm{AdS}_{5}$ :

$$
\ell^{2}=\frac{12}{V_{0}} .
$$

We have defined the following ratio of the $\beta$-function coefficients,

$$
b=\frac{b_{1}}{b_{0}^{2}} .
$$

This ratio has the interesting property that it is left invariant under a multiplicative renormalization of the coupling constant. This follows from the Gell-Mann-Low equation and explained in detail in appendix A.

On the other hand, the asymptotic form of the dilaton to subleading order is,

$$
b_{0} \lambda=\frac{\ell}{u}+\left(b-\frac{4}{9}\right) \log u+\cdots
$$

One can also easily obtain the asymptotic form of the Ricci scalar and find that $R \rightarrow-20 / \ell^{2}$ : We have the $A d S_{5}$ geometry in the UV and the subleading term in (4.11) can be viewed as a perturbation on $\mathrm{AdS}_{5}$.

We also present the same asymptotics in the conformal $r$-coordinate system:

$$
\begin{gathered}
d s^{2}=\frac{\ell^{2}}{r^{2}}\left(1+\frac{8}{9} \frac{1}{\log (r \Lambda)}-\frac{8}{9} b \frac{\log (-\log (r \Lambda))}{\log (r \Lambda)^{2}}+\cdots\right)\left(d x^{i} d x_{i}+d r^{2}\right) \\
\lambda=-\frac{1}{b_{0} \log (r \Lambda)}+\frac{b}{b_{0}} \frac{\log (-\log (r \Lambda))}{(\log (r \Lambda))^{2}}+\cdots
\end{gathered}
$$

where $\ell$ is the AdS radius and $\Lambda$ is an integration constant, that corresponds to the perturbative QCD scale in the dual theory.

We remark that the leading and the subleading terms in the metric, (4.11), depend neither on the energy-radius coefficients $f_{i}$, nor on the particular $\beta$-function coefficients $b_{i}$. Therefore the first terms in (4.11) are universal and solely follow from the requirement of asymptotic freedom. However, the subleading term of the metric already introduces the invariant QCD scale $\Lambda$. Of course the information on the $\beta$-function is encoded in the leading term of the dilaton solution.

\subsection{Confinement and the IR geometry}

Given the geometry, a natural question to ask is whether or not this geometry describes quark confinement in the IR. On the gauge theory side, a criterion for confinement is that the Wilson loop operator exhibits an area law behavior at large distances. This corresponds to an asymptotic linear potential between two external quarks. In the $5 \mathrm{~d}$ dual this criterion can be tested by calculating the classical action 
of a fundamental string world-sheet that extends to the Wilson loop at the $A d S_{5}$ boundary, as argued in 34, 35].

A detailed analysis with a classification of all the confining backgrounds is presented in [20]. Here we would like to discuss the qualitative features of the set-up by focusing on one class of asymptotics:

$$
X(\lambda)=-\frac{1}{2}\left[1+\frac{a}{\log \lambda}+\ldots\right], \quad \lambda \rightarrow \infty
$$

The asymptotics of the geometry can be obtained using the definition (3.4):

$$
A=A_{0}+\frac{1}{3} \int_{0}^{\lambda} \frac{d \lambda^{\prime}}{\lambda^{\prime} X\left(\lambda^{\prime}\right)},
$$

and $\Phi$ from (3.5). In the conformal variable, one obtains the following IR asymptotics $($ as $r \rightarrow \infty)$

$$
A=-C r^{\alpha}+\text { subleading, }
$$

where $C$ is an integration constant and we have defined,

$$
\alpha=\frac{3}{3-4 a} \text {. }
$$

The dilaton behaves as,

$$
\Phi=\frac{3}{2} C r^{\alpha}+\text { subleading }
$$

It is shown in [20] that these backgrounds confine for $\alpha \geq 1$. To see this, one needs to take into account the subleading term in (4.21). In the borderline case, $\alpha=1$, the geometry is interesting and well-known. In the string frame the metric asymptotically becomes,

$$
d s^{2} \rightarrow d x^{2}+\frac{4}{9} d \Phi^{2}, \quad \Phi \rightarrow \infty .
$$

This is the linear-dilaton background. The Ricci scalar in the string frame therefore vanishes in the IR. In the Einstein frame, the metric has a singularity at a finite value $u=u_{0}$ (corresponding to $r \rightarrow \infty$. There, the scale factor $\exp [A]$ shrinks to zero. This is due to the fact that $e^{\Phi}$ and therefore the gauge coupling diverge at that point. The stringy corrections to the geometry in this regime can be neglected as in the string frame the background is known to be exact to all orders in $\alpha^{\prime}$.

The linear dilaton asymptotics are the borderline of confining backgrounds. The spectrum of mesons and glueballs is obtained by considering the fluctuations of the geometry. Although exhibiting a mass gap, the spectrum is partly continuous for $\alpha=1$. Therefore, we discard the borderline case $\alpha=1(a=0)$ for physical reasons. In the case $\alpha>1$ however, the spectrum turns out to be discrete.

For $\alpha>1$ (or $a>0$ ) the linear dilaton background in (4.22) is modified into the following asymptotic metric:

$$
d s^{2} \rightarrow \Phi^{\frac{4 a}{3}} d x^{2}+\frac{4}{9} \Phi^{-\frac{4 a}{3}} d \Phi^{2}, \quad \Phi \rightarrow \infty
$$


The Ricci scalar in the string frame reads asymptotically ,

$$
R_{s} \sim-4 a(7 a-3) \Phi^{\frac{4}{3} a-2} .
$$

We observe that for the confining backgrounds $\alpha>1$ (or $0<a<3 / 4$ ) the string frame Ricci scalar vanishes in the IR. This implies that the singularity in the string frame is only due to the diverging dilaton. This is a good type of singularity where the string moving in this background feels very small curvature corrections.

The effect of $\alpha^{\prime}$ corrections to the confinement criteria is an important question to which we turn. Fortunately, as happens with "good" holographic singularities, the low-lying fluctuations have an effective potential that shields them from the singularity. Therefore, the $\alpha^{\prime}$ corrections are under control in this case, see [20].

Let us finally note the behavior of the dilaton potential and the various geometric invariants in this region. The dilaton potential follows from (3.8):

$$
V \approx \frac{3}{4} V_{0} \lambda^{\frac{4}{3}}(\log \lambda)^{\frac{\alpha-1}{\alpha}}\left(1+\mathcal{O}\left(\frac{1}{\log \lambda}\right)\right) .
$$

Similarly one obtains the following asymptotics for the geometric invariants in the Einstein frame,

$$
E \sim R \sim(\partial \Phi)^{2} \sim \lambda^{\frac{4}{3}}(\log \lambda)^{\frac{\alpha-1}{\alpha}} .
$$

We see that this is the linear dilaton background with logarithmic corrections. In the $\alpha=1$ case the leading behavior of the geometry is exactly that of the linear dilaton background.

\section{Examples of background geometries}

In this section we present examples of full background geometries that exhibit the desired UV and IR asymptotics discussed in the previous two sections. The first is an example showing asymptotic freedom in the UV and confinement in the IR. For comparison, we present an example which is asymptotically free in the UV, but has a conformal fixed point in the IR. Finally, in appendix $\mathbb{E}$ we present a third example which is asymptotically free in the UV and has a $\beta$-function that asymptotes to zero in the IR with an exponential tail.

\subsection{Standard QCD type two-loop $\beta$-function}

We start from the "exact" $\beta$-function:

$$
\beta(\lambda)=-\frac{3 b_{0} \lambda^{2}}{3+2 b_{0} \lambda}-\frac{\left(2 b_{0}^{2}+3 b_{1}\right) \lambda^{3}}{3\left(1+\lambda^{2}\right)\left[1+\frac{\left(2 b_{0}^{2}+3 b_{1}\right)}{18 a} \log \left(1+\lambda^{2}\right)\right]} .
$$

with $b_{0,1}>0, a>0$. This expression is chosen so that one has the desired asymptotics in the UV and IR and such that there are no poles or branch cuts in $\lambda$. In particular at 


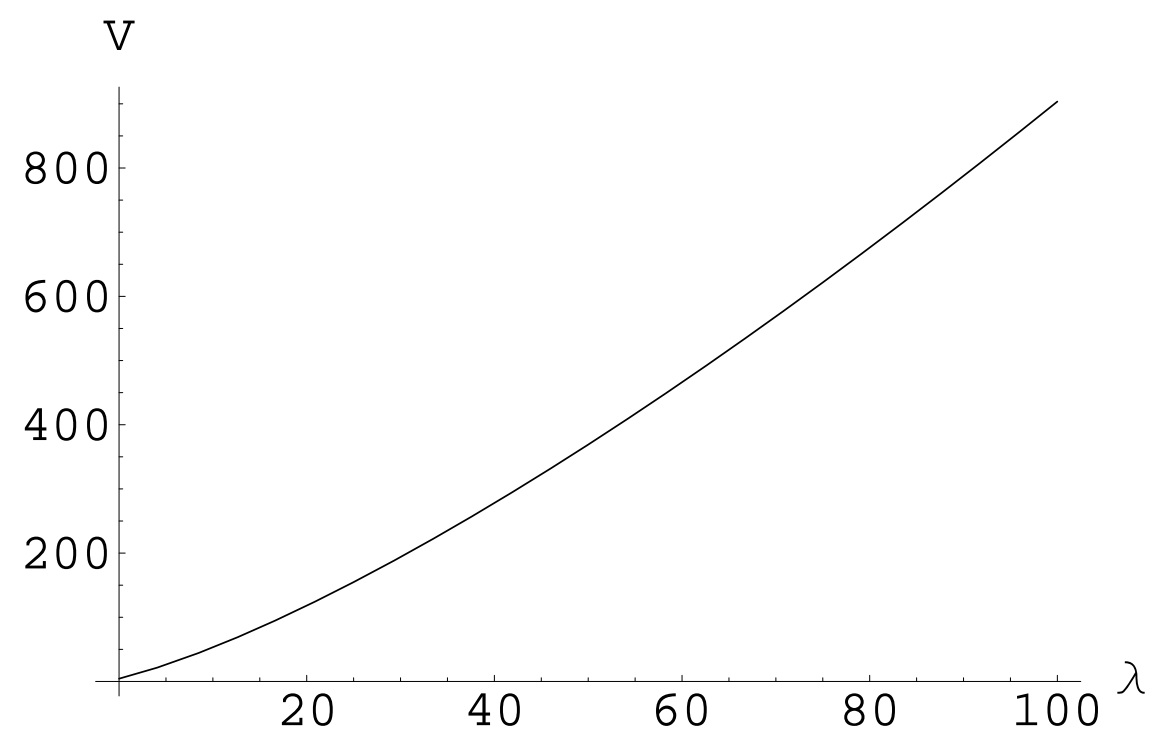

Figure 1: Dilaton potential as a function of $\lambda$ for the solution studied in section 5.1.

weak coupling it reproduces the QCD result to two loops, $\beta=-b_{0} \lambda^{2}-b_{1} \lambda^{3}+\mathcal{O}\left(\lambda^{4}\right)$. At strong coupling it behaves as $\beta=-\frac{3}{2} \lambda+3 a \frac{\lambda}{\log \lambda}+\cdots$ as required by (3.21) and (4.17). It also avoids unwanted singularities or branch points at finite values of $\lambda$.

According to (3.8) the dilaton potential that corresponds to this specific $\beta$ function is,

$$
V(\lambda)=V_{0}\left(1-X(\lambda)^{2}\right)\left(3+2 b_{0} \lambda\right)^{\frac{4}{3}}\left(18 a+\left(2 b_{0}^{2}+3 b_{1}\right) \log \left(1+\lambda^{2}\right)\right)^{\frac{8 a}{3}}
$$

where the phase-space variable $X(\lambda)$ is given by $\beta(\lambda) / 3 \lambda$. The equation that determines the running of $\lambda$ follows from, (3.5):

$$
\lambda^{\prime}=\frac{3}{\ell} X(\lambda)\left(3+2 b_{0}\right)^{\frac{2}{3}}\left(18 a+\left(2 b_{0}^{2}+3 b_{1}\right) \log \left(1+\lambda^{2}\right)\right)^{\frac{4 a}{3}} .
$$

We give the plot of the potential in fig 1. The solution of (5.3) as a function of $u$, with the initial condition $\lambda(0)=0$ is shown in fig 2 .

We see from the figures that $\lambda$ diverges in the IR at the singularity $u_{0}$.

The associated geometry can be derived as follows. The metric in the string frame is,

$$
d s^{2}=e^{2 A+\frac{4}{3} \Phi} d x^{2}+e^{\frac{4}{3} \Phi} d r^{2}=\lambda^{\frac{4}{3}}\left(e^{2 A} d x^{2}+\left(\lambda^{\prime}\right)^{-2} d \lambda^{2}\right)
$$

The metric function $A$ is obtained as a function of $\lambda$ from (4.18). Unfortunately, it is not possible to express this integral in terms of elementary functions. The Einstein frame metric is obtained from (5.4) by multiplying with $\lambda^{-4 / 3}$. The asymptotics of this geometry are discussed in the previous section. 


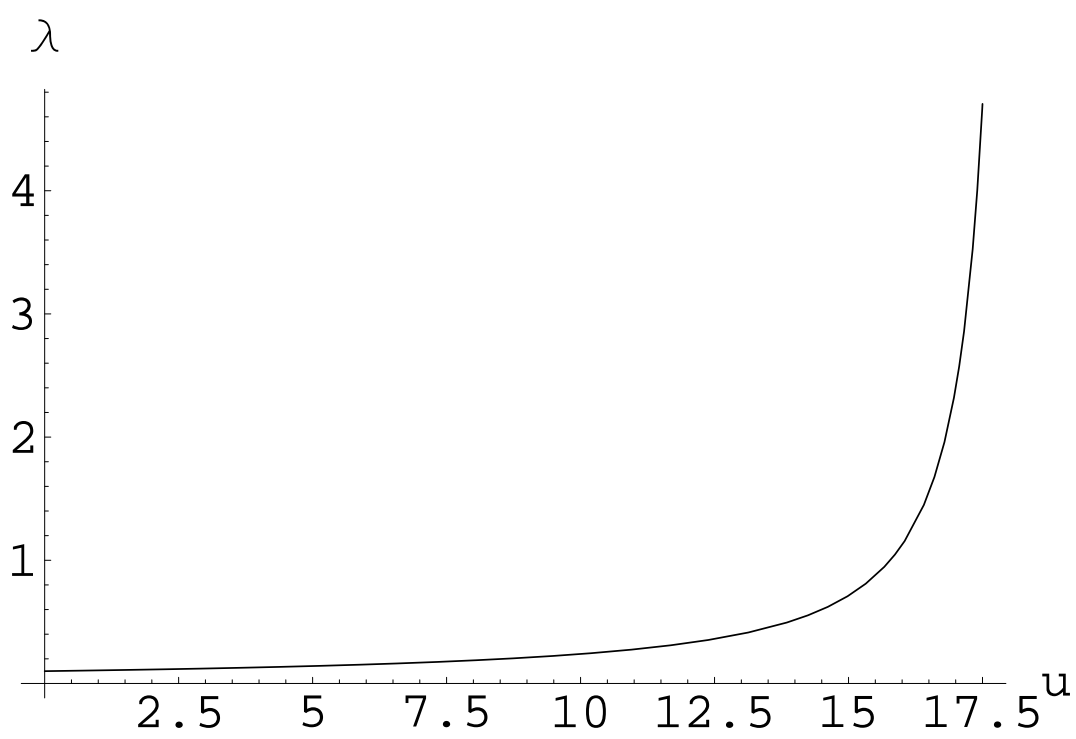

Figure 2: The running of the coupling constant. It diverges at $u_{0}$.

One can also easily determine certain invariants of the geometry. The Ricci scalar is given by (3.9) and $R=-3 / 2 E$. In the Einstein frame, one obtains,

$$
R=-\frac{3}{2} V_{0}\left(\frac{5}{2}-4 X(\lambda)^{2}\right)\left(3+2 b_{0} \lambda\right)^{\frac{4}{3}}\left(18 a+\left(2 b_{0}^{2}+3 b_{1}\right) \log \left(1+\lambda^{2}\right)\right)^{\frac{8 a}{3}} .
$$

The asymptotic behavior of the various geometric invariants in this geometry are also presented in the previous two sections.

\section{$5.2 \beta$-function with an IR fixed point}

We choose an exact $\beta$-function as

$$
\beta(\lambda)=\frac{-b_{0} \lambda^{2}+\bar{b}_{1} \lambda^{3}}{1+\frac{2 b_{0}}{3} \lambda-\frac{2 \bar{b}_{1}}{3} \lambda^{2}}
$$

with

$$
\bar{b}_{1}=b_{1}-2 b_{0}^{2} / 3>0 .
$$

In perturbation theory we have $\beta=-b_{0} \lambda^{2}+b_{1} \lambda^{3}+\mathcal{O}\left(\lambda^{4}\right)$ with $b_{0,1}>0$.

According to $(3.8)$ the potential for this specific $\beta$-function is,

$V(\lambda)=\frac{V_{0}}{\left(\lambda_{+}\right)^{a_{+}}\left(-\lambda_{-}\right)^{a_{-}}}\left(\left(\lambda_{+}-\lambda\right)^{2}\left(\lambda-\lambda_{-}\right)^{2}-\frac{1}{4} \lambda^{2}\left(\lambda_{0}-\lambda\right)^{2}\right)\left(\lambda_{+}-\lambda\right)^{a_{+}-2}\left(\lambda_{-} \lambda_{-}\right)^{a_{-}-2}$.

Here we defined the following quantities. $\lambda_{0}=b_{0} / \bar{b}_{1}$ is the value of the coupling constant at the IR fixed point. $\lambda_{ \pm}$is defined as:

$$
\lambda_{ \pm}=\frac{\lambda_{0}}{2} \pm \frac{\sqrt{\lambda_{0}^{2}+\frac{6}{\overline{b_{1}}}}}{2}
$$




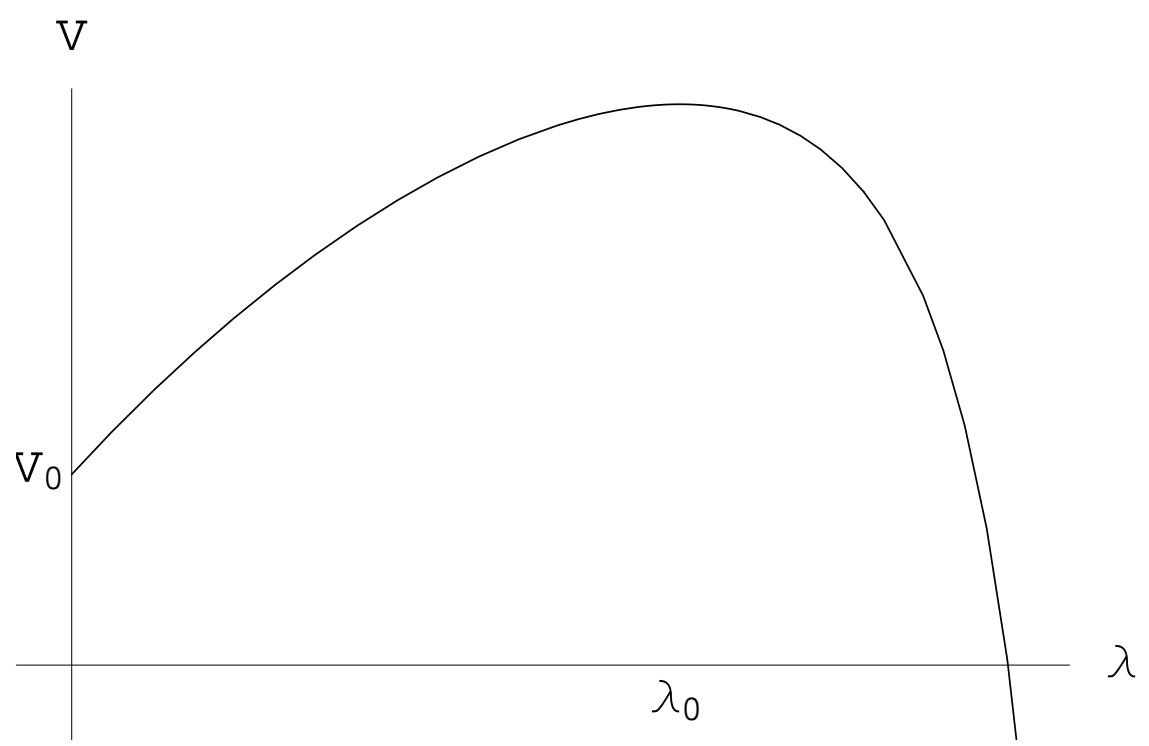

Figure 3: Dilaton potential as a function of $\lambda$ when there is an IR fixed point. We have set $V_{0}=b_{0}=b_{1}=1$ for simplicity.

and the exponents are,

$$
a_{ \pm}=-\mp \frac{4}{3} \frac{\lambda_{\mp}}{\lambda_{+}-\lambda_{-}}
$$

We note that along the RG flow, the range of $\lambda$ is such that, $\lambda_{-}<0<\lambda<\lambda_{0}<\lambda_{+}$.

We give the plot of the potential in fig. 3. The RG flow is from $\lambda=0$ in the UV toward $\lambda=\lambda_{0}$ in the IR. As we discussed in section 4 the UV geometry is AdS with the radius given by eq. (4.12). As fig. 3 suggests, the IR end of the potential is also AdS.

Below we present the details of the IR geometry. The IR AdS radius is obtained by taking the $\lambda \rightarrow \lambda_{0}$ limit in the potential, (5.8):

$$
\ell_{I R}=2 \sqrt{\frac{3}{V_{f}}}, \quad V_{f}=V_{0}\left(\frac{-\lambda_{-}}{\lambda_{+}}\right)^{a_{+}-a_{-}} .
$$

We may deduce from (5.11) that $V_{f} / V_{0}>1$. Therefore, the c-theorem of 36] is obeyed for this holographic RG flow.

The equation that determines the running of $\lambda$ follows from, (3.5) as,

$$
\lambda^{\prime}=\frac{\left(3 V_{0}\right)^{\frac{1}{2}}}{2} \lambda^{2}\left(\lambda_{0}-\lambda\right)\left(\lambda_{+}-\lambda\right)^{\frac{a_{+}}{2}-1}\left(\lambda-\lambda_{-}\right)^{\frac{a_{-}}{2}-1} .
$$

The solution of (5.12) as a function of $u$, with the initial condition $\lambda(0)=0$ is shown in fig 4 . We observe that, $\lambda$ flows into a non-trivial fixed point at low energies. The value of the coupling constant at the fixed point is $\lambda_{0}=b_{0} / \bar{b}_{1}$. As $\lambda$ increases from 


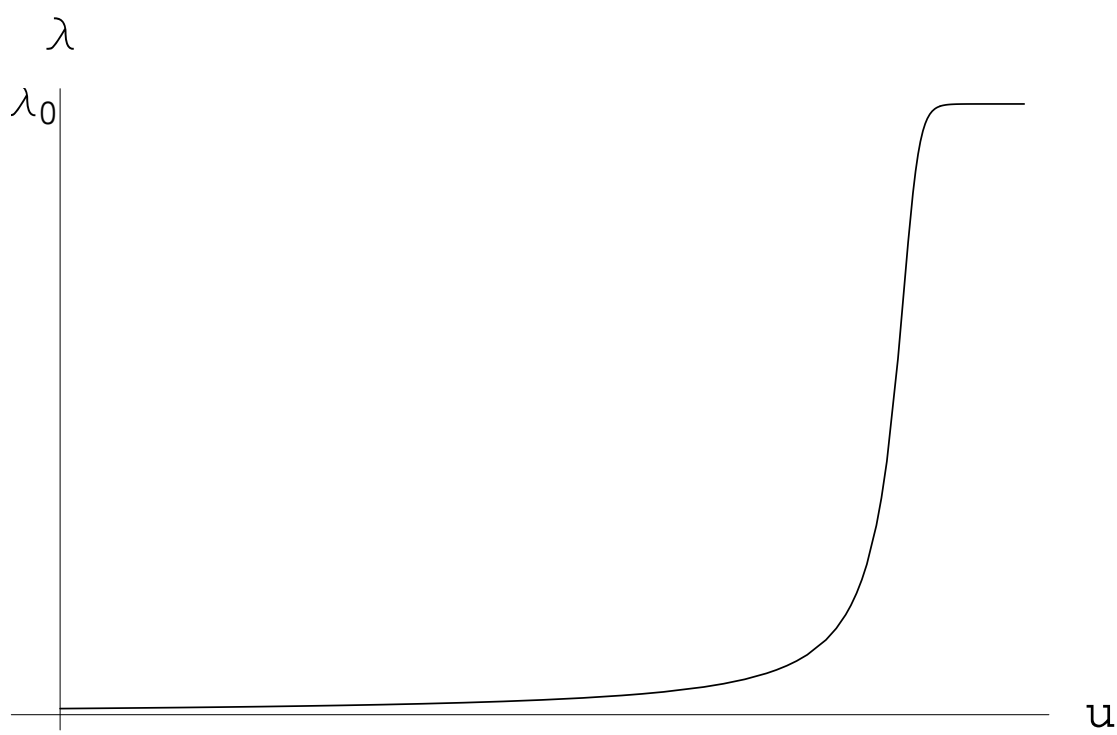

Figure 4: Running of the coupling constant in the Banks-Zaks case. We have set $V_{0}=$ $b_{0}=b_{1}=1$.

0 to $\lambda_{0}$ during the RG flow, one has the inequality $\lambda_{-}<0<\lambda<\lambda_{0}<\lambda_{+}$in this range.

The metric in the string frame (5.4) is obtained by using (4.18) and (3.5) as,

$$
d s^{2}=e^{2 A_{0}+\frac{2}{b_{0} \lambda}} \lambda^{-\frac{2 \bar{b}_{1}}{b_{0}^{2}}}\left(\lambda_{0}-\lambda\right)^{\frac{2 \bar{b}_{1}}{b_{0}^{2}}} d x^{2}+\frac{16}{V_{0}}\left(\lambda_{0}-\lambda\right)^{-2}\left(\lambda_{+}-\lambda\right)^{-a_{+}}\left(\lambda-\lambda_{-}\right)^{-a_{-}} \frac{d \lambda^{2}}{\lambda^{\frac{8}{3}}} .
$$

One can also easily determine certain invariants of the geometry. The Ricci scalar is given by (3.9) and $R=-3 / 2 E$. In the Einstein frame we obtain,

$$
R=-\frac{3}{4} V_{0}\left(5\left(\lambda-\lambda_{-}\right)^{2}\left(\lambda_{+}-\lambda\right)^{2}-2 \lambda^{2}\left(\lambda_{0}-\lambda\right)^{2}\right)\left(\lambda-\lambda_{-}\right)^{a_{-}}\left(\lambda_{+}-\lambda\right)^{a_{+}}
$$

Note that $R$ goes to a constant in the IR, i.e. as $\lambda \rightarrow \lambda_{0}$. This is in accordance with the presence of the fixed point in the IR. Similarly the invariant $(\partial \Phi)^{2}$ follows from (5.13) as,

$$
(\partial \Phi)^{2}=g^{\lambda \lambda}\left(\partial_{\lambda} \Phi\right)^{2}=\frac{V_{0}}{16} \lambda^{2}\left(\lambda-\lambda_{-}\right)^{a_{-}}\left(\lambda_{+}-\lambda\right)^{a_{+}}\left(\lambda_{0}-\lambda\right)^{2},
$$

which vanishes in the IR as required from the conformality.

The IR asymptotics of the geometry can be obtained by solving the equations (3.6) and (3.5) near $\lambda_{0}$ (that corresponds to $u=\infty$ ). We present the geometry in the conformal coordinate, by performing the change of variable (2.15). One finds,

$$
d s^{2}=\frac{l_{I R}^{2}}{r^{2}}\left(1+\frac{1}{r \Lambda}\right)^{-2}\left(d x^{2}+d r^{2}\right)
$$


near $r \rightarrow \infty$. Here $\Lambda$ is an integration constant of equation (3.5). The gauge theory interpretation of $\Lambda$ is the energy scale below which the conformality in the IR sets in.

We may determine the exact form of the quark potential and check that it indeed has the conformal form in the IR. We find the following relation between the quarkanti-quark potential and the distance in the IR asymptotics,

$$
E=\frac{C\left[\lambda_{0}, \bar{b}_{1}\right]^{2}}{L},
$$

with the constant $C$ is given by,

$$
C\left[\lambda_{0}, b_{1}\right]=\frac{2 \bar{b}_{1}^{-\frac{1}{2}}}{\sqrt{3 V_{0}}} \frac{\lambda_{0}^{\frac{5}{3}}}{\left(\lambda_{+}-\lambda_{0}\right)^{\frac{a_{+}}{2}}\left(\lambda_{0}-\lambda_{-}\right)^{\frac{a_{-}}{2}}} .
$$

This exhibits conformal behavior.

Of course, as the IR theory is conformal, we do not expect this model to be a good for description of pure YM type theories. We have presented it however, for illustration purposes.

\section{Fluctuations near the boundary}

The general geometry discussed in the previous section is asymptotically AdS near the boundary, $u \rightarrow \infty$. It is important to study the fluctuations of the fields on this background. According to the standard rules of AdS/CFT, near the boundary, these fluctuations should correspond either to sources or expectation values of the corresponding operators of the gauge theory. In what follows, we compute the UV asymptotics of the fluctuations keeping the subleading terms as well. According to the principles of AdS/CFT these subleading terms should give information on the anomalous dimensions of the operators dual to the fluctuations.

For the case of a 5D geometry coupled to one scalar field the analysis of the fluctuations have been carried out in [37, 38] (see also [39] and [40]). We are interested in the fluctuations of the metric, the dilaton and the axion. The metric and the dilaton can be decomposed into $4 \mathrm{D}$ traceless-transverse tensor $h_{\mu \nu}$, two transverse gauge fields $A_{\mu}$ and $V_{\mu}$ and 5 scalar fields.

The tensor modes obeys the usual $5 \mathrm{D}$ wave equation:

$$
\square_{5} h_{\mu \nu}=0,
$$

where $\square_{5}$ is the scalar Laplacian on the 5 D space-time ${ }^{19}$ :

$$
\square_{5}=e^{-2 A}\left(\frac{d^{2}}{d r^{2}}+3 \frac{d A}{d r} \frac{d}{d r}+\square_{4}\right) .
$$

\footnotetext{
${ }^{19}$ In this section we use the conformal coordinate system, eq. (2.13)
} 
From (6.1), it is clear that the non-normalizable solution for $h_{\mu \nu}$ sources a dimension 4 operator. It is the energy-momentum tensor of the $4 \mathrm{D}$ boundary theory. Also it can be seen that the vector fluctuations can completely be gauged away by using 5 D diffeomorphisms. ${ }^{20}$ Thus, in what follows we focus on the interesting case of the scalar fluctuations.

\subsection{Dilaton fluctuations}

As discussed in detail e.g. in [40], there is a single diffeomorphism invariant combination among the 5 scalar fluctuations:

$$
\zeta=\psi-\frac{1}{3 X} \chi
$$

Here $X$ is the variable defined in (3.4), $\psi$ is related to the trace of the metric fluctuation and $\chi$ is the fluctuation of the dilaton. We note from (4.2) that, for any asymptotically-free boundary theory, $X$ approaches zero in the UV. As a result, close to the boundary, $\psi$ and $\chi$ decouple from each other and $\zeta$ reduces to the dilaton fluctuation. In the conformal coordinate system (2.13), it obeys the following equation:

$$
\ddot{\zeta}+\left(3 \dot{A}+2 \frac{\dot{X}}{X}\right) \dot{\zeta}-k^{2} \zeta=0,
$$

where $k^{\mu}$ is the $4 \mathrm{D}$ momentum.

It is interesting to comment on the correspondence of the gauge invariant scalar fluctuation $\zeta$, with the Yang-Mills operators. Indeed, it corresponds to the "dressed" $\operatorname{Tr}\left[F^{2}\right]$ operator

$$
\zeta \leftrightarrow \beta(\lambda) \operatorname{Tr}\left[F^{2}\right]
$$

which is known to be RG invariant (see for example [41]). The invariance of $\zeta$ under infinitesimal $r$ reparametrizations, guarantees that it corresponds to a RG-invariant operator.

Using the asymptotic forms of the dilaton and the metric in (4.15) and (4.16) close to the boundary, (6.4) becomes (in units where $\ell=1$ ),

$$
\ddot{\zeta}-\frac{1}{r}\left(3+\frac{2}{\log r \Lambda}+\frac{C}{\log ^{2} r \Lambda}-2 b \frac{\log (-\log r \Lambda)}{\log ^{2} r \Lambda}\right) \dot{\zeta}-k^{2} \zeta=0,
$$

where $b$ is defined in (4.13) and the coefficient $C$ is given by,

$$
C=\frac{4}{3}+2 b\left(2-\log b_{0}\right) .
$$

The corresponding fluctuation equation in the pure $\mathrm{AdS}_{5}$ geometry is given by,

$$
\ddot{\zeta}-\frac{3}{r} \dot{\zeta}-k^{2} \zeta=0
$$

\footnotetext{
${ }^{20}$ This is strictly true only for the $4 \mathrm{D}$ massive modes, 40], which is the case we are interested in.
} 
There are two independent solutions to (6.6), one is normalizable and the other is non-normalizable. The asymptotic expansion of the non-normalizable solution reads,

$$
\begin{aligned}
\zeta_{\text {source }}= & 1-\frac{(k r)^{2}}{4}\left[1-\frac{1}{\log r \Lambda}+\frac{2-C}{2 \log ^{2} r \Lambda}+b \frac{\log (-\log r \Lambda)}{\log ^{2} r \Lambda}+\cdots\right] \\
& +\frac{(k r)^{4}}{64}\left[1-\frac{3}{2 \log r \Lambda}+\frac{7-3 C}{4 \log ^{2} r \Lambda}+\frac{3 b \log (-\log r \Lambda)}{2 \log ^{2} r \Lambda}+\cdots\right]+\mathcal{O}\left(r^{6}\right)(.6 .9)
\end{aligned}
$$

According to the standard rules of the AdS/CFT correspondence this solution is dual to a source term for a gauge-invariant operator of engineering dimension 4 and parity +1 in the boundary gauge theory. As argued above such an operator is $\beta(\lambda) \operatorname{Tr} F^{2}$.

The other solution of (6.6) is normalizable near the boundary and it has the following asymptotic expansion:

$\zeta_{v e v}=(k r)^{4}\left[\log ^{2} r \Lambda+2 b \log r \Lambda \log (-\log r \Lambda)+\left(2 b-C-\frac{1}{2}\right) \log r \Lambda+\cdots\right]+\mathcal{O}\left(r^{6}\right)$

This solution corresponds to a VEV for $\operatorname{Tr} F^{2}$, namely a gluon condensate.

\subsection{Axion fluctuations}

The other scalar in the geometry is the axion. Its background value is non-zero if $\theta_{U V} \neq 0$ and is obtained by solving

$$
\frac{1}{\sqrt{g}} \partial_{\mu}\left[Z(\Phi) \sqrt{g} g^{\mu \nu} \partial_{\nu}\right] a=0
$$

in the Einstein frame. $Z$ is an "effective" potential that resums higher-order corrections due to the five form, as explained in appendix B.1. The background solution $a(r)$ describes the RG running of the QCD $\theta$ angle. As the axion is suppressed by extra powers of $1 / N_{c}$, 28, this equation can be solved in the background already determined by the metric-dilaton equations. We analyze it in detail in 20] where the vacuum condensate of $\operatorname{Tr}[F \wedge F]$ is calculated. Here we note that since the action for the axion is quadratic, its background value does not affect its fluctuations that are dual to the $\operatorname{Tr}[F \wedge F]$ operator in QCD.

The equation for the axion fluctuations follow from (2.4) and we obtain ,

$$
\ddot{\alpha}+\left(3 \dot{A}+2 \frac{\dot{Z}}{Z}\right) \dot{\alpha}-k^{2} \alpha=0
$$

The non-normalizable axion fluctuation corresponds to a source term for the operator $\operatorname{Tr} F \wedge F$ and the normalizable solution is dual to a VEV for the same operator, $\langle\operatorname{Tr} F \wedge F\rangle$, namely an axial glueball condensate. This will be further determined in 20 . 


\section{Acknowledgments}

It is a pleasure to thank G. Bali, B. Bringoltz, R. Casero, L. Giusti, D. K. Hong, K. Intriligator, M. Luscher, J. Mas, H.B. Meyer, C. Morningstar, V. Niarchos, C. Nunez, H. Panagopoulos, I. Papadimitriou, S. Pal, A. Paredes, G. Poliscastro, F. Sannino, C. Skenderis, M. Shifman, E. Shuryak, S. J. Sin, J. Sonnenschein, M. Teper, J. Troost, A. Vainshtein, G. Veneziano, A. Vladikas, and especially F. Nitti for useful discussions and insights. UG is supported by European Commission Marie Curie Postdoctoral Fellowships, under contract MEIF-CT-2006-039962. This work was also partially supported by INTAS grant, 03-51-6346, RTN contracts MRTN-

CT-2004-005104 and MRTN-CT-2004-503369, CNRS PICS \# 3059 and 3747 , and by a European Union Excellence Grant, MEXT-CT-2003-509661. 


\section{APPENDIX}

\section{A. Asymptotic expansions in the (UV) weak-coupling regime}

In this appendix we will carefully analyze the weak coupling expansion in the UV regime of the space, near the boundary. We start from the equations of motion in the conformal frame as described in section 2 ,

$$
d s^{2}=e^{2 A(r)}\left[d r^{2}+\eta_{i j} d x^{i} d x^{j}\right] \quad, \quad E_{r r}=6 \dot{A}^{2} \quad, \quad E_{i j}=3\left[\ddot{A}+\dot{A}^{2}\right] \eta_{i j}
$$

where we use the mostly plus convention for the metric. The two scalar invariants of the metric up to quadratic order are

$$
E_{\mu}{ }^{\mu}=-\frac{3}{2} R=6 e^{-2 A}\left[2 \ddot{A}+3 \dot{A}^{2}\right] \quad, \quad E_{\mu \nu} E^{\mu \nu}=36 e^{-4 A}\left[\ddot{A}^{2}+2 \ddot{A} \dot{A}^{2}+2 \dot{A}^{4}\right]
$$

In this ansatz the equations (2.11) become

$$
\begin{gathered}
12 \dot{A}^{2}-\frac{4}{3} \dot{\phi}^{2}-e^{2 A} V=0 \quad, \quad 6 \ddot{A}+6 \dot{A}^{2}+\frac{4}{3} \dot{\phi}^{2}-e^{2 A} V=0 \\
\ddot{\phi}+3 \dot{A} \dot{\phi}+\frac{3}{8} e^{2 A} V^{\prime}=0 \quad, \quad V^{\prime}=\frac{d V(\phi)}{d \phi}
\end{gathered}
$$

To continue further we define the 't Hooft coupling and its inverse as usual

$$
\lambda=N_{c} e^{\phi} \quad, \quad \alpha_{s}=\frac{1}{\lambda} \quad, \quad \dot{\phi}=-\frac{\dot{\alpha_{s}}}{\alpha_{s}} \quad, \quad \ddot{\phi}=-\frac{\ddot{\alpha_{s}}}{\alpha_{s}}+\frac{\dot{\alpha}_{s}^{2}}{\alpha_{s}^{2}}
$$

(A.3) and (A.4) become

$$
-\frac{\ddot{\alpha_{s}}}{\alpha_{s}}+\frac{\dot{\alpha}_{s}^{2}}{\alpha_{s}^{2}}-3 \dot{A} \frac{\dot{\alpha}_{s}}{\alpha_{s}}+\frac{3}{8} e^{2 A} V^{\prime}=0 \quad, \quad 12 \dot{A}^{2}-\frac{4}{3} \frac{\dot{\alpha}_{s}{ }^{2}}{\alpha_{s}^{2}}-e^{2 A} V=0
$$

We now change variables to

$$
r=e^{-t} \quad, \quad e^{A(t)}=e^{t} d(t)
$$

where $t$ is essentially the log of the energy in the perturbative region. The equations become

$$
\begin{gathered}
-\frac{\alpha_{s}^{\prime \prime}}{\alpha_{s}}-4 \frac{\alpha_{s}^{\prime}}{\alpha_{s}}+\frac{\alpha_{s}^{\prime 2}}{\alpha_{s}^{2}}-3 \frac{\alpha_{s}^{\prime}}{\alpha_{s}} \frac{d^{\prime}}{d}+\frac{3}{8} d^{2} V^{\prime}=0 \\
12\left(1+\frac{d^{\prime}}{d}\right)^{2}-\frac{4}{3} \frac{\alpha_{s}^{\prime 2}}{\alpha_{s}^{2}}-d^{2} V=0
\end{gathered}
$$

where we now use primes for $t$ derivatives. 
We assume a regular power expansion for the potential $V=V_{0}+V_{1} \lambda+V_{2} \lambda^{2}+V_{3} \lambda^{3}+V_{4} \lambda^{4}+\cdots \quad, \quad V^{\prime}=V_{1} \lambda+2 V_{2} \lambda^{2}+3 V_{3} \lambda^{3}+4 V_{4} \lambda^{4}+\cdots$

We now substitute for the inverse coupling

$$
\begin{gathered}
\frac{1}{\lambda}=\alpha_{s}=L-\frac{b_{1}}{b_{0}} \log L+\frac{b_{1}^{2}}{b_{0}^{2}} \frac{\log L}{L}+\left(\frac{b_{1}^{2}}{b_{0}^{2}}+\frac{b_{2}}{b_{0}}\right) \frac{1}{L}+\frac{b_{1}^{3}}{2 b_{0}^{3}} \frac{\log ^{2} L}{L^{2}}+ \\
+\frac{b_{1} b_{2}}{b_{0}^{2}} \frac{\log L}{L^{2}}+\left[\frac{b_{3}}{2 b_{0}}-\frac{b_{1}^{3}}{2 b_{0}^{3}}\right] \frac{1}{L^{2}}+\mathcal{O}\left(\frac{1}{L^{3}}\right) \\
L=a_{0}+b_{0} t=-b_{0} \log (r \Lambda) \quad, \quad L^{\prime}=b_{0}
\end{gathered}
$$

The expression above is taylored so that coupling satisfies the standard RG equation

$$
\lambda^{\prime}=-b_{0} \lambda^{2}+b_{1} \lambda^{3}+b_{2} \lambda^{4}+b_{3} \lambda^{5} \ldots \quad, \quad \alpha_{s}^{\prime}=b_{0}-\frac{b_{1}}{\alpha_{s}}-\frac{b_{2}}{\alpha_{s}^{2}}-\frac{b_{3}}{\alpha_{s}^{3}}+\cdots
$$

where $\Lambda$ is the RG invariant scale of QCD.

We may rewrite now equations (A.8), A.9) as

$$
\frac{d^{\prime}}{d}=\frac{1}{8} \frac{\alpha_{s}}{\alpha_{s}^{\prime}} d^{2} V^{\prime}-\frac{4}{3}-\frac{1}{3} \frac{\alpha_{s}^{\prime \prime}}{\alpha_{s}^{\prime}}+\frac{1}{3} \frac{\alpha_{s}^{\prime}}{\alpha_{s}} \quad, \quad \frac{d^{\prime}}{d}=-1+\sqrt{\frac{d^{2} V}{12}+\frac{1}{9} \frac{\alpha_{s}^{\prime 2}}{\alpha_{s}^{2}}}
$$

so that

$$
\frac{1}{8} \frac{\alpha_{s}}{\alpha_{s}^{\prime}} d^{2} V^{\prime}-\frac{1}{3}-\frac{1}{3} \frac{\alpha_{s}^{\prime \prime}}{\alpha_{s}^{\prime}}+\frac{1}{3} \frac{\alpha_{s}^{\prime}}{\alpha_{s}}=\sqrt{\frac{d^{2} V}{12}+\frac{1}{9} \frac{\alpha_{s}^{\prime 2}}{\alpha_{s}^{2}}}
$$

Note that only one branch of the square root is relevant. We expand the potential in inverse powers of logs to obtain ${ }^{21}$

$$
\begin{gathered}
V=V_{0}+\frac{V_{1}}{L}+\frac{V_{2}}{L^{2}}+\frac{b_{1}}{b_{0}} V_{1} \frac{\log L}{L^{2}}+\left(V_{3}-\frac{b_{2}}{b_{0}} V_{1}-\frac{b_{1}^{2}}{b_{0}^{2}} V_{1}\right) \frac{1}{L^{3}}+ \\
+\frac{b_{1}^{2}}{b_{0}^{2}} V_{1} \frac{(\log L)^{2}}{L^{3}}+\left(2 \frac{b_{1}}{b_{0}} V_{2}-\frac{b_{1}^{2}}{b_{0}^{2}} V_{1}\right) \frac{\log L}{L^{3}}+\mathcal{O}\left(\frac{1}{L^{4}}\right) \\
\frac{\alpha_{s}}{\alpha_{s}^{\prime}} V^{\prime}=\frac{V_{1}}{b_{0}}+\left(\frac{2 V_{2}}{b_{0}}+\frac{b_{1} V_{1}}{b_{0}^{2}}\right) \frac{1}{L}+\left(\frac{3 V_{3}}{b_{0}}+\frac{b_{2} V_{1}}{b_{0}^{2}}\right) \frac{1}{L^{2}}+\left(\frac{2 b_{1} V_{2}}{b_{0}^{2}}+\frac{b_{1}^{2} V_{1}}{b_{0}^{3}}\right) \frac{1+\log L}{L^{2}}+\mathcal{O}\left(\frac{1}{L^{3}}\right)
\end{gathered}
$$

From (A.15) we obtain

$$
\frac{3}{8} d^{2}=\frac{V+\frac{\alpha_{s}}{\alpha_{s}^{\prime}} V^{\prime}\left(1+\frac{\alpha_{s}^{\prime \prime}}{\alpha_{s}^{\prime}}-\frac{\alpha_{s}^{\prime}}{\alpha_{s}}\right)}{\left(\frac{\alpha_{s}}{\alpha_{s}^{\prime}} V^{\prime}\right)^{2}} \pm
$$

\footnotetext{
${ }^{21}$ We thank Shesansu Pal for pointing out errors in the following two equations in the first version of this paper.
} 


$$
\pm \frac{\sqrt{\left[V+\frac{\alpha_{s}}{\alpha_{s}^{\prime}} V^{\prime}\left(1+\frac{\alpha_{s}^{\prime \prime}}{\alpha_{s}^{\prime}}-\frac{\alpha_{s}^{\prime}}{\alpha_{s}}\right)\right]^{2}-\left(1+\frac{\alpha_{s}^{\prime \prime}}{\alpha_{s}^{\prime}}-2 \frac{\alpha_{s}^{\prime}}{\alpha_{s}}\right)\left(1+\frac{\alpha_{s}^{\prime \prime}}{\alpha_{s}^{\prime}}\right)\left(\frac{\alpha_{s}}{\alpha_{s}^{\prime}} V^{\prime}\right)^{2}}}{\left(\frac{\alpha_{s}}{\alpha_{s}^{\prime}} V^{\prime}\right)^{2}}
$$

Using

$$
\begin{gathered}
V+\frac{\alpha_{s}}{\alpha_{s}^{\prime}} V^{\prime}\left(1+\frac{\alpha_{s}^{\prime \prime}}{\alpha_{s}^{\prime}}-\frac{\alpha_{s}^{\prime}}{\alpha_{s}}\right)=V_{0}+\frac{V_{1}}{b_{0}}+\left(\frac{2 V_{2}}{b_{0}}+\frac{b_{1} V_{1}}{b_{0}^{2}}\right) \frac{1}{L}+\left(\frac{2 b_{1} V_{2}}{b_{0}^{2}}+\frac{b_{1}^{2} V_{1}}{b_{0}^{3}}\right) \frac{\log L}{L^{2}} \\
+\left(\frac{3 V_{3}}{b_{0}}+\frac{2 b_{1} V_{2}}{b_{0}^{2}}-V_{2}-\frac{b_{2} V_{1}}{b_{0}^{2}}+\frac{b_{1}^{2} V_{1}}{b_{0}^{3}}\right) \frac{1}{L^{2}}+\mathcal{O}\left(\frac{1}{L^{3}}\right)
\end{gathered}
$$

we obtain

$$
\begin{aligned}
& \frac{3}{8} d^{2}=\frac{b_{0}}{V_{1}}\left(1+\frac{b_{0} V_{0}}{V_{1}}\right)-\frac{\left(2 b_{0} V_{0}+V_{1}\right)\left(b_{1} V_{1}+2 b_{0} V_{2}\right)}{V_{1}^{3} L}-\frac{b_{1}\left(2 b_{0} V_{0}+V_{1}\right)\left(b_{1} V_{1}+2 b_{0} V_{2}\right) \log L}{b_{0} V_{1}^{3} L^{2}} \\
& +\left[-\frac{3 b_{0}\left(2 b_{0} V_{0}+V_{1}\right) V_{3}}{V_{1}^{3} L^{2}}+\frac{4 b_{0}\left(3 b_{0} V_{0}+V_{1}\right) V_{2}^{2}}{V_{1}^{4} L^{2}}-\frac{b_{0}^{2} V_{2}}{V_{1}^{2} L^{2}}+\frac{2 b_{1}\left(4 b_{0} V_{0}+V_{1}\right) V_{2}}{V_{1}^{3} L^{2}}\right. \\
& \left.\quad+\frac{b_{2}\left(2 b_{0} V_{0}+V_{1}\right)}{V_{1}^{2} L^{2}}+\frac{b_{1}\left(b_{1} V_{0}+b_{0} V_{1}\right)}{V_{1}^{2} L^{2}}\right] \pm \\
& \pm\left[\frac{b_{0}^{2}}{V_{1}^{2}} \sqrt{V_{0}\left(V_{0}+2 \frac{V_{1}}{b_{0}}\right)}-\sqrt{b_{0}} \frac{\left(2 b_{0} b_{1} V_{0}^{2} V_{1}+3 b_{1} V_{0} V_{1}^{2}-b_{0} V_{1}^{3}+4 b_{0}^{2} V_{0}^{2} V_{2}+6 b_{0} V_{0} V_{1} V_{2}\right)}{V_{1}^{3} \sqrt{V_{0}\left(2 V_{1}+b_{0} V_{0}\right)} L}+\mathcal{O}\left(\frac{1}{L^{2}}\right)\right]
\end{aligned}
$$

Compatibility to leading order with the first of (A.14) implies

$$
V_{1}=\frac{8}{9} b_{0} V_{0}
$$

for the plus sign. The minus sign does not lead to a consistent solution. Therefore to leading order

$$
\frac{3}{8} d^{2} V_{0}=\frac{b_{0} V_{0}}{V_{1}}\left(1+\frac{b_{0} V_{0}}{V_{1}}\right)+\left(\frac{b_{0} V_{0}}{V_{1}}\right)^{2} \sqrt{1+2 \frac{V_{1}}{b_{0} V_{0}}}=\frac{9}{2} \Rightarrow d^{2} V_{0}=12
$$

Using (A.22), (A.21) becomes

$$
\begin{gathered}
\frac{3}{8} V_{0} d^{2}=\frac{9}{2}+\frac{3\left(b_{0}^{2} V_{0}-12 b_{1} V_{0}-27 V_{2}\right)}{5 b_{0} V_{0} L}-\frac{3 b_{1}\left(-b_{0}^{2} V_{0}+12 b_{1} V_{0}+27 V_{2}\right)}{5 b_{0}^{2} V_{0} L^{2}} \\
-\frac{\left(64 b_{0}^{4}-756 b_{0}^{2} b_{1}-1944 b_{1}^{2}-5400 b_{0} b_{2}\right) V_{0}^{2}+2349 b_{0}^{2} V_{0} V_{2}-20898 b_{1} V_{0} V_{2}-37179 V_{2}^{2}+18225 b_{0} V_{0} V_{3}}{750 b_{0}^{2} V_{0}^{2} L^{2}} \\
+\mathcal{O}\left(\frac{1}{L^{3}}\right)
\end{gathered}
$$

We may calculate from (A.24)

$$
\frac{d^{\prime}}{d}=-\frac{b_{0}^{2} V_{0}-12 b_{1} V_{0}-27 V_{2}}{15 V_{0} L^{2}}-\frac{2 b_{1}\left(b_{0}^{2} V_{0}-12 b_{1} V_{0}-27 V_{2}\right) \log L}{15 b_{0} V_{0} L^{3}}
$$




$$
+\frac{94 b_{0}^{4}-1251 b_{0}^{2} b_{1}-324 b_{1}^{2}-5400 b_{0} b_{2}}{3375 b_{0} L^{3}}+\frac{9\left(3 b_{0}^{2}-31 b_{1}\right) V_{2}}{125 b_{0} V_{0} L^{3}}-\frac{567 V_{2}^{2}}{125 b_{0} v_{0}^{2} L^{3}}+\frac{27 V_{3}}{5 V_{0} L^{3}}+\cdots
$$

Compatibility with the first of (A.14) implies

$$
\begin{gathered}
\frac{V_{2}}{V_{0}}=\frac{23 b_{0}^{2}-36 b_{1}}{3^{4}}, \frac{V_{3}}{V_{0}}=-2 \frac{324 b_{2}+124 b_{0}^{3}+189 b_{0} b_{1}}{3^{7}} \\
\frac{V_{4}}{V_{0}}=\frac{3176 b_{0}^{4}+7236 b_{0}^{2} b_{1}-243 b_{1}^{2}-810 b_{0} b_{2}-4374 b_{3}}{3^{9}}
\end{gathered}
$$

Using this we finally obtain for the metric

$$
\begin{gathered}
\frac{V_{0}}{12} d^{2} \equiv \frac{d^{2}}{\ell^{2}}=1-\frac{8 b_{0}}{3^{2} L}+\frac{4\left(26 b_{0}^{2}+9 b_{1}\right)}{3^{4} L^{2}}-\frac{8 b_{1} \log L}{3^{2} L^{2}}-\frac{8}{3^{2}} \frac{b_{1}^{2}}{b_{0}} \frac{\log ^{2} L}{L^{3}} \\
+\frac{16 b_{1}\left(13 b_{0}^{2}+9 b_{1}\right)}{3^{4} b_{0}} \frac{\log L}{L^{3}}-\frac{8}{3^{7} b_{0}}\left(698 b_{0}^{4}+594 b_{0}^{2} b_{1}-243 b_{1}^{2}-324 b_{0} b_{2}\right) \frac{1}{L^{3}}+\mathcal{O}\left(\frac{1}{L^{4}}\right)
\end{gathered}
$$

Therefore to next to leading order the Poicaré metric near the boundary is

$$
d s^{2}=\left[1+\frac{8}{3^{2} \log r \Lambda}+\frac{4\left(26+9 \frac{b_{1}}{b_{0}^{2}}-18 \frac{b_{1}}{b_{0}^{2}} \log \left(b_{0} \log \frac{1}{r \Lambda}\right)\right)}{3^{4} \log ^{2} r \Lambda}+\mathcal{O}\left(\frac{\log ^{2} \log r \Lambda}{\log ^{3} r \Lambda}\right)\right] \frac{\ell^{2}}{r^{2}}\left(d r^{2}+d \vec{x}^{2}\right)
$$

We observe that the first non-trivial correction is independent of $b_{0}$. This is related to the fact that as we have no unambiguous identification of the normalization of the gauge coupling, the $\beta$-function coefficients we are using can be changed as

$$
b_{n} \rightarrow a^{n+1} b_{n}
$$

From (A.27) we observe that $d$ is invariant up to the shift of the logarithms, $\log L=$ $\log \left(b_{0} \log \frac{1}{r \Lambda}\right)$ since it depends only on $b_{1} / b_{0}^{2}$ and $b_{2} / b_{0}^{3}$. It is a non-trivial statement that a change of scale in the coupling as in (A.29), in (A.27) can be absorbed into a change of the scale $\Lambda$ as well as the non-universal $\beta$-function coefficients $b_{n>1}$.

The potential in the UV regime can be therefore expanded in terms of the overall AdS scale as well as the $\beta$-function coefficients to obtain

$$
V=\frac{12}{\ell^{2}}\left[1+\frac{8}{9}\left(b_{0} \lambda\right)+\frac{23-36 \frac{b_{1}}{b_{0}^{2}}}{3^{4}}\left(b_{0} \lambda\right)^{2}-2 \frac{324 \frac{b_{2}}{b_{0}^{3}}+124+189 \frac{b_{1}}{b_{0}^{2}}}{3^{7}}\left(b_{0} \lambda\right)^{3}+\mathcal{O}\left(\lambda^{4}\right)\right]
$$

\section{A.1 Scalar curvature invariants in the UV regime}

We may now evaluate the basic scalar invariants of the background in the weak coupling (UV) regime:

$$
V=V_{0}\left[1+\frac{8}{9} \frac{b_{0}}{L}+\frac{23 b_{0}^{2}}{81 L^{2}}+\frac{8 b_{1} \log L}{9 L^{2}}+\mathcal{O}\left(\frac{1}{L^{3}}\right)\right]
$$




$$
\begin{aligned}
& (\partial \phi)^{2}=e^{-2 A} \frac{\dot{\alpha}_{s}^{2}}{\alpha_{s}^{2}}=\frac{r^{2}}{d^{2}} \frac{\dot{\alpha}_{s}{ }^{2}}{\alpha_{s}^{2}}=\frac{1}{d^{2}} \frac{\alpha_{s}^{\prime 2}}{\alpha_{s}^{2}}=V_{0}\left[\frac{b_{0}}{12 L}+\frac{b_{0} \log L}{12 L^{2}}+\frac{8 b_{0}^{2}-9 b_{1}}{108 L^{2}}+\mathcal{O}\left(\frac{1}{L^{3}}\right)\right] \\
& R=\frac{4}{3}(\partial \phi)^{2}-\frac{5}{3} V
\end{aligned}
$$

It is obvious that all invariants are regular in the UV, $(L \rightarrow \infty)$. All higher curvature invariants are also regular as they are polynomial functions of

$$
e^{-2 A} \dot{A}^{2}=\frac{1}{9}(\partial \phi)^{2}+\frac{1}{12} V \quad, \quad e^{-2 A} \ddot{A}=-\frac{1}{3}(\partial \phi)^{2}+\frac{1}{12} V
$$

\section{A.2 The two-loop $\beta$-function coefficients of pure gauge theory}

As a final point we quote the scheme-independent $\beta$-function coefficients for QCD, $b_{0}, b_{1}$.

With $N_{f}$ (non-chiral) flavors in the fundamental, the $\beta$-function reads

$$
\beta(g)=-\frac{g^{3}}{(4 \pi)^{2}}\left\{\frac{11}{3} N_{c}-\frac{2}{3} N_{f}\right\}-\frac{g^{5}}{(4 \pi)^{4}}\left\{\frac{34}{3} N_{c}^{2}-\frac{N_{f}}{N_{c}}\left[\frac{13}{3} N_{c}^{2}-1\right]\right\}+\cdots
$$

For the the 't Hooft coupling, and with $\frac{N_{f}}{N_{c}} \rightarrow x$ we obtain

$$
\lambda \equiv g^{2} N_{c} \quad, \quad \dot{\lambda}=-\frac{2}{3}\left[\frac{(11-2 x)}{(4 \pi)^{2}} \lambda^{2}+\frac{(34-13 x)}{(4 \pi)^{4}} \lambda^{3}+\cdots\right]
$$

from where we obtain

$$
b_{0}=\frac{2}{3} \frac{(11-2 x)}{(4 \pi)^{2}} \quad, \quad \frac{b_{1}}{b_{0}^{2}}=-\frac{3}{2} \frac{(34-13 x)}{(11-2 x)^{2}}
$$

In this paper, $x=0$ and therefore $\frac{b_{1}}{b_{0}^{2}}=-\frac{3 \cdot 34}{2 \cdot 121} \simeq 0.42$.

\section{B. General potentials at string tree level}

Extra terms in the dilaton potential can arise from higher $\alpha^{\prime}$ corrections proportional to the field strength of the RR four-form. We parametrize these corrections in the string frame as

$$
S_{F}=-\frac{M^{3}}{2 \ell_{s}^{2}} \int d^{5} x \sqrt{g} e^{-2 \phi} K\left(e^{2 \phi} y\right)
$$

where

$$
K(y) \equiv-2 \delta c+\sum_{n=1}^{\infty} \frac{a_{n}}{n} y^{n} \quad, \quad y=\frac{\ell_{s}^{2}\left(F_{5}\right)^{2}}{5 !} \quad, \quad a_{1}=1
$$

and we assumed the simplest type of contraction for simplicity. The contribution for general type of contractions yield qualitatively the same result upon modifying the coefficients, $a_{n}$. Therefore we will assume that (B.1) captures all such corrections. 
Passing to the Einstein frame we obtain,

$$
S_{F}^{E}=-\frac{M^{3}}{2 \ell_{s}^{2}} \int d^{5} x \sqrt{g} e^{\frac{4}{3} \phi} K\left(e^{-\frac{14}{3} \phi} y\right)
$$

The equations of motion are

$$
\nabla^{\mu_{1}}\left[e^{-\frac{10}{3} \phi} K^{\prime}\left(e^{-\frac{14}{3} \phi} y\right) F_{\mu_{1} \mu_{2} \cdots \mu_{5}}\right]=0
$$

with solution

$$
K^{\prime}\left(e^{-\frac{14}{3} \phi} y\right) F_{\mu_{1} \mu_{2} \cdots \mu_{5}}=N_{c} e^{\frac{10}{3} \phi} E_{\mu_{1} \mu_{2} \cdots \mu_{5}}
$$

where $N_{c}$ will now be non-linearly related to the number of color branes. By squaring we obtain the consistency conditions

$$
y\left[K^{\prime}\left(e^{-\frac{14}{3} \phi} y\right)\right]^{2}=-N_{c}^{2} e^{\frac{20}{3} \phi} \quad, \quad\left(K^{\prime}\right)^{2} \frac{F_{\mu \nu}^{2}}{4 !}=-\frac{N_{c}^{2}}{\ell_{s}^{2}} e^{\frac{20}{3} \phi} g_{\mu \nu}
$$

so that

$$
F_{\mu \nu}^{2}=\frac{F^{2}}{5} g_{\mu \nu}
$$

We now compute the contribution of the five-form to the stress tensor

$$
T_{\mu \nu}^{F}=\frac{1}{\sqrt{g}} \frac{\delta S_{F}^{E}}{\delta g^{\mu \nu}}=-\frac{M^{3}}{2}\left[e^{-\frac{10}{3} \phi} K^{\prime} \frac{F_{\mu \nu}^{2}}{4 !}-\frac{g_{\mu \nu}}{2 \ell_{s}^{2}} e^{\frac{4}{3} \phi} K\right]=\frac{M^{3} e^{\frac{4}{3} \phi}}{2 \ell_{s}^{2}}\left[-e^{-\frac{14}{3} \phi} y K^{\prime}+\frac{1}{2} K\right] g_{\mu \nu}
$$

We may therefore substitute the action of the five form $S_{F}^{E}$ with

$$
\begin{gathered}
\hat{S}_{F}^{E}=\frac{M^{3}}{\ell_{s}^{2}} \int d^{5} x \sqrt{g} V_{E}(\phi) \\
V_{E}(\phi)=e^{\frac{4}{3} \phi}\left[e^{-\frac{14}{3} \phi} X(\phi) K^{\prime}\left(e^{-\frac{14}{3} \phi} X(\phi)\right)-\frac{1}{2} K\left(e^{-\frac{14}{3} \phi} X(\phi)\right)\right]
\end{gathered}
$$

In the formulae above $y=X(\phi)$ is a solution of $(\mathbb{B} .6)$.

We redefine

$$
\zeta=e^{-\frac{14}{3} \phi} y
$$

Then the relevant equations become

$$
\zeta\left[K^{\prime}(\zeta)\right]^{2}=-N_{c}^{2} e^{2 \phi} \quad, \quad V_{E}=e^{\frac{4}{3} \phi}\left[\zeta K^{\prime}(\zeta)-\frac{1}{2} K(\zeta)\right]
$$

We return to the $\sigma$-model frame in order to estimate the large- $N_{c}$ dependence

$$
S_{\sigma}=M^{3} \int d^{5} x \sqrt{g} e^{-2 \phi}\left[R+4(\partial \phi)^{2}+\frac{1}{\ell_{s}^{2}} V_{\sigma}(\phi)\right] \quad, \quad V_{\sigma}(\phi)=\left[\zeta K^{\prime}(\zeta)-\frac{1}{2} K(\zeta)\right]
$$


If we define the 't Hooft coupling as

$$
\lambda \equiv N_{c} e^{\phi}
$$

then from (B.12), $\zeta$ is a function of $\lambda$. The $\sigma$-model frame action (B.13) becomes

$$
S_{\sigma}=N_{c}^{2} M^{3} \int d^{5} x \sqrt{g} \frac{1}{\lambda^{2}}\left[R+4 \frac{(\partial \lambda)^{2}}{\lambda^{2}}+\frac{1}{\ell_{s}^{2}} V_{\sigma}(\lambda)\right]
$$

We observe the $\mathcal{O}\left(N_{c}^{2}\right)$ dependence expected from the sphere action as well as the fact that there is a non-trivial potential for the 't Hooft coupling.

For small $\zeta$ we can calculate the potential perturbatively:

$$
K(\zeta)=-2 \delta c+\zeta+\frac{1}{2} a_{2} \zeta^{2}+\mathcal{O}\left(\zeta^{3}\right) \quad, \quad \zeta K^{\prime}(\zeta)=\zeta+a_{2} \zeta^{2}+\mathcal{O}\left(\zeta^{3}\right)
$$

to obtain

$$
\zeta=-\lambda^{2}-2 a_{2} \lambda^{4}+\mathcal{O}\left(\lambda^{6}\right) \quad, \quad V_{\sigma}=-\frac{1}{2}\left(-2 \delta c+\lambda^{2}+\frac{a_{2}}{2} \lambda^{4}+\mathcal{O}\left(\lambda^{6}\right)\right)
$$

For a DBI-like example : $K(y)=2 \sqrt{1+y}$ we obtain

$$
\zeta=-\frac{\lambda^{2}}{1+\lambda^{2}} \quad, \quad V(\lambda)=-\sqrt{1+\lambda^{2}}
$$

It is obvious from the above analysis that $V(\lambda)$ has an infinite series of terms, that, after solving the equations of motion of the four-form, are independent from $\alpha^{\prime}$ except an overall (universal) dependence in front. Therefore the higher-order in $\alpha^{\prime}$ corrections to the four-form are equivalent to a (leading) order in $\alpha^{\prime}$ potential. Surprisingly, this potential has a regular expansion at weak YM coupling.

This analysis obviously generalizes to the kinetic terms of the dilaton and graviton. The general action is of the form

$$
S=\int d^{5} x \sqrt{g} e^{-2 \phi}\left[V R+4 V_{1}(\partial \phi)^{2}+V_{2}\right]
$$

The functions $V, V_{1,2}$ depend on $e^{2 \phi} y$ with $y$ given in (B.2), and summarize the higher $\alpha^{\prime}$-corrections of the four-form. Going through the same procedure as above, it can be shown that this is equivalent with integrating out the four-form, and multiplying the kinetic terms with appropriate series in the exponential of the dilaton as in the standard potential. Moreover, higher derivative terms proportional to powers of the curvature as well as the dilaton derivatives will be generated.

\section{B.1 Corrections to the axion terms}

The axion $a$, dual to the instanton density has a special position among the supergravity fields. Its shift symmetry is protected, and its special normalization in the 
YM theory implies that its contributions are suppressed by a power of $1 / N_{c}^{2}$, due to the fact that the dual variable in YM is an angle, 28]. This is reflected in the fact that in string theory, $a$ is a RR field and therefore has suppressed dilaton dependence. The leading term in the effective action is $\int \sqrt{g}(\partial a)^{2}$ in the string frame, and becomes

$$
\int \sqrt{g} e^{2 \phi}(\partial a)^{2}=\frac{1}{N_{c}^{2}} \int \sqrt{g} \lambda^{2}(\partial a)^{2}
$$

in the Einstein frame where in the second equality we indicated the suppression of the $\theta$-induced vacuum energy.

We now consider the higher terms in the $\alpha^{\prime}$ expansion that involve the 5 -form field strength. In analogy with the previous section we may write them in the string frame $a^{22}$

$$
S_{F, a}=-\frac{M^{3}}{2 \ell_{s}^{2}} \int d^{5} x \sqrt{g} e^{-2 \phi}\left[K_{1}\left(e^{2 \phi} y\right)+K_{2}\left(e^{2 \phi} y\right) e^{2 \phi}(\partial a)^{2}\right],
$$

where

$$
K_{1}(y) \equiv-2 \delta c \sum_{n=1}^{\infty} \frac{a_{n}}{n} y^{n} \quad, \quad K_{2}(y) \equiv 1+\sum_{n=1}^{\infty} \frac{b_{n}}{n} y^{n} \quad, \quad y=\frac{\ell_{s}^{2}\left(F_{5}\right)^{2}}{5 !} \quad, \quad a_{1}=1
$$

Passing to the Einstein frame we obtain,

$$
S_{F}^{E}=-\frac{M^{3}}{2 \ell_{s}^{2}} \int d^{5} x \sqrt{g} e^{\frac{4}{3} \phi}\left[K_{1}\left(e^{-\frac{14}{3} \phi} y\right)+K_{2}\left(e^{-\frac{14}{3} \phi} y\right) e^{\frac{2}{3} \phi}(\partial a)^{2}\right]
$$

The equations of motion for the 5-form are

$$
\nabla^{\mu_{1}}\left[e^{-\frac{10}{3} \phi} K_{1}^{\prime}\left(e^{-\frac{14}{3} \phi} y\right)+e^{-\frac{8}{3} \phi} K_{2}^{\prime}\left(e^{-\frac{14}{3} \phi} y\right)(\partial a)^{2}\right] F_{\mu_{1} \mu_{2} \cdots \mu_{5}}=0
$$

with solution

$$
\left[K_{1}^{\prime}\left(e^{-\frac{14}{3} \phi} y\right)+e^{\frac{2}{3} \phi} K_{2}^{\prime}\left(e^{-\frac{14}{3} \phi} y\right)(\partial a)^{2}\right] F_{\mu_{1} \mu_{2} \cdots \mu_{5}}=N_{c} e^{\frac{10}{3} \phi} E_{\mu_{1} \mu_{2} \cdots \mu_{5}}
$$

By squaring we obtain the consistency conditions

$$
y\left[K_{1}^{\prime}\left(e^{-\frac{14}{3} \phi} y\right)+e^{\frac{2}{3} \phi} K_{2}^{\prime}\left(e^{-\frac{14}{3} \phi} y\right)(\partial a)^{2}\right]^{2}=-N_{c}^{2} e^{\frac{20}{3} \phi}
$$

and

$$
\left[K_{1}^{\prime}+e^{\frac{2}{3} \phi} K_{2}^{\prime}(\partial a)^{2}\right]^{2} \frac{F_{\mu \nu}^{2}}{4 !}=-\frac{N_{c}^{2}}{\ell_{s}^{2}} e^{\frac{20}{3} \phi} g_{\mu \nu}
$$

so that

$$
F_{\mu \nu}^{2}=\frac{F^{2}}{5} g_{\mu \nu}
$$

\footnotetext{
${ }^{22}$ Higher terms like $(\partial a)^{4}$ are suppressed by extra powers of $N_{c}$ and we do not need to consider them here.
} 
We now compute the contribution of the five-form to the stress tensor

$$
\begin{gathered}
-\frac{2}{M^{3}} T_{\mu \nu}^{F}=-\frac{2}{M^{3}} \frac{1}{\sqrt{g}} \frac{\delta S_{F}^{E}}{\delta g^{\mu \nu}}=e^{-\frac{10}{3} \phi}\left[K_{1}^{\prime}+e^{\frac{2}{3} \phi} K_{2}^{\prime}(\partial a)^{2}\right] \frac{F_{\mu \nu}^{2}}{4 !}+\frac{e^{2 \phi}}{\ell_{s}^{2}} K_{2} \partial_{\mu} a \partial_{\nu} a- \\
-\frac{g_{\mu \nu}}{2 \ell_{s}^{2}} e^{\frac{4}{3} \phi}\left[K_{1}+e^{\frac{2}{3} \phi} K_{2}(\partial a)^{2}\right] \\
=\frac{e^{\frac{4}{3} \phi}}{\ell_{s}^{2}}\left[e^{-\frac{14}{3} \phi} y\left[K_{1}^{\prime}+e^{\frac{2}{3} \phi} K_{2}^{\prime}(\partial a)^{2}\right]-\frac{1}{2}\left[K_{1}+e^{\frac{2}{3} \phi} K_{2}(\partial a)^{2}\right]\right] g_{\mu \nu}+\frac{e^{2 \phi}}{\ell_{s}^{2}} K_{2} \partial_{\mu} a \partial_{\nu} a
\end{gathered}
$$

The axion equation also reads

$$
\nabla^{\mu}\left[e^{2 \phi} K_{2} \nabla_{\mu} a\right]=0
$$

We redefine

$$
\zeta=e^{-\frac{14}{3} \phi} y \quad, \quad \eta=e^{\frac{2}{3} \phi}(\partial a)^{2}
$$

and $(\mathbb{B . 2 6})$ becomes

$$
\zeta\left[K_{1}^{\prime}(\zeta)+K_{2}^{\prime}(\zeta) \eta\right]^{2}=-N_{c}^{2} e^{2 \phi}
$$

The dual action which gives the same equations of motion is

$$
\tilde{S}^{E}=\frac{M^{3}}{\ell_{s}^{2}} \int d^{5} x \sqrt{g} e^{\frac{4}{3} \phi}\left[\zeta\left(K_{1}^{\prime}(\zeta)+\eta K_{2}^{\prime}(\zeta)\right)-\frac{1}{2}\left(K_{1}(\zeta)+\eta K_{2}(\zeta)\right)\right]
$$

In the action above, $\zeta(\lambda, \eta)$ is a solution of (B.33). In the string frame the dual action becomes

$$
\tilde{S}_{\sigma}=\frac{M^{3}}{\ell_{s}^{2}} \int d^{5} x \sqrt{g} e^{-2 \phi}\left[\zeta\left(K_{1}^{\prime}(\zeta)+\tilde{\eta} K_{2}^{\prime}(\zeta)\right)-\frac{1}{2}\left(K_{1}(\zeta)+\tilde{\eta} K_{2}(\zeta)\right)\right]
$$

with $\tilde{\eta}=e^{2 \phi}(\partial a)^{2}$. We must separate the kinetic term of the axion from the higher derivative terms that appear because $\zeta$ depends non-trivially on $\eta$. This turns out to be in the Einstein frame

$$
\tilde{S}_{\text {axion-linear }}^{E}=-\frac{M^{3}}{2 \ell_{s}^{2}} \int d^{5} x \sqrt{g} e^{2 \phi} K_{2}\left(\zeta^{*}\right)(\partial a)^{2}
$$

where now $\zeta^{*}$ is a solution $\zeta K_{1}^{\prime}(\zeta)=-N_{c}^{2} e^{2 \phi}$.

We finish this section by giving the most general dual action involving the five form

$$
S=\int d^{5} x \sqrt{g} e^{-2 \phi} Z\left(e^{2 \phi} y, z_{i}\right)
$$

where $Z$ is an arbitrary function and $z_{i}$ are scalar invariants of other fields, $R, R^{2}, R_{\mu \nu} R^{\mu \nu}$, $\left.(\partial \phi)^{2}, e^{2 \phi}(\partial a)^{2}\right)$ etc.

The dual action is given by the Legendre transform

$$
\tilde{S}=\int d^{5} x \sqrt{g}\left[\zeta \partial_{\zeta} Z\left(\zeta, z_{i}\right)-\frac{1}{2} Z\left(\zeta, z_{i}\right)\right]
$$

where $\zeta$ satisfies

$$
\zeta\left(\partial_{\zeta} Z\left(\zeta, z_{i}\right)\right)^{2}=-\lambda^{2}
$$




\section{B.2 Corrections to the gauge coupling constant identification}

Consider now a probe $\mathrm{D}_{3}$ brane and the coupling of the kinetic gauge field terms

$$
S_{D_{3}}=\frac{T_{3}}{\ell_{s}^{4}} \int d^{4} x \sqrt{\hat{g}} e^{-\phi} Z\left(e^{2 \phi} y\right) \operatorname{Tr}\left[F^{2}\right], Z(y)=1+\sum_{n=1}^{\infty} \frac{c_{n}}{n} y^{n}, y=\frac{\ell_{s}^{2}\left(F_{5}\right)^{2}}{5 !}
$$

where $T_{3}$ is dimensionless and $Z(y)$ summarizes higher-order couplings of the five form on the D-brane (arising from disk diagrams). Going to the Einstein frame and substituting from B.11,B.12 we obtain for the gauge coupling constant

$$
g_{Y M}^{2}=\frac{e^{\phi}}{Z(\zeta)}
$$

Therefore, these corrections, although without derivatives, are due to the higher $\alpha^{\prime}$ corrections on the branes. A similar argument indicates that the tension of possible flavor branes obtains similar types of corrections.

\section{B.3 Other higher derivative corrections at the tree level}

After dualizing the five form, there remain true higher-derivative corrections associated to curvatures and derivatives of the dilaton. We will indicate here how such corrections can be instrumental in effectively generating a constant term in the dilaton potential, a fact that we have assumed in this paper in order to simplify our problem.

To give the idea, we will focus on the tree level, string frame effective action without dilatonic kinetic terms and considered only as a function of the scalar curvature (for simplicity)

$$
S=\int d^{5} x \sqrt{g} e^{-2 \phi} f\left(\ell_{s}^{2} R, e^{2 \phi} y\right)
$$

where we are using the same definition of $y$ as in (B.2) and $f$ is a function with a regular series expansion around $R=y=0$. The equations of motion are

$$
\begin{gathered}
\ell_{s}^{2} f_{R} R_{\mu \nu}-\frac{1}{2} g_{\mu \nu} f+e^{2 \phi}\left[g_{\mu \nu} \square-\nabla_{\mu} \nabla_{\nu}\right]\left(e^{-2 \phi} f\right)=0 \quad, \quad f_{R} \equiv \frac{\delta f}{\delta R} \\
\nabla^{\mu}\left[f_{y} F_{\mu \nu_{1} \cdots \nu_{4}}\right]=0 \quad, \quad f_{y} \equiv \frac{\delta f}{\delta y}
\end{gathered}
$$

These equations admit an $A d S_{5}$ solution,

$$
R_{\mu \nu}=-\frac{4}{\ell_{A d S}^{2}} g_{\mu \nu}
$$

with $\phi=$ constant and

$$
f_{y} F_{\nu_{1} \cdots \nu_{5}}=\frac{N_{c}}{\ell_{s}} E_{\nu_{1} \cdots \nu_{5}} \quad \rightarrow \quad y f_{y}^{2}\left[x, e^{2 \phi} y\right]=-N_{c}^{2}
$$


where we defined

$$
x=-20 \frac{\ell_{s}^{2}}{\ell^{2}}
$$

The metric equation then becomes

$$
2 x f_{R}\left[x, e^{2 \phi} y\right]+5 f\left[x, e^{2 \phi} y\right]=0
$$

and typically the two algebraic equations are expected to have a solution. A simple example involves

$$
f=e^{2 \phi} y+\ell_{s}^{2} R+\mu \ell_{s}^{4} R^{2}
$$

which generates an $A d S_{5}$ space with

$$
\frac{\ell^{2}}{\ell_{s}^{2}}=\frac{9 \mu}{10\left(7 \pm \sqrt{49+180 \mu \lambda^{2}}\right)} \quad, \quad \lambda \equiv e^{\phi} N_{c}
$$

In particular, a solution exists for $\lambda=0$ and it is

$$
\frac{\ell^{2}}{\ell_{s}^{2}}=\frac{9 \mu}{140}
$$

It is not difficult to verify that allowing now the dilaton to run logarithmically in the UV, so that $\lambda \rightarrow 0$ as $r \rightarrow 0$ is a small (subleading) perturbation in the $\alpha^{\prime}$ expansion, generating a solution that logarithmically asymptotes to the AdS solution above. In particular, the kinetic terms of the dilaton that have been neglected will be suppressed by large inverse logs, and the same applies to the power series in the t' Hooft coupling $N_{c} e^{\phi}$. We will not pursue this avenue further here, leaving it for a future investigation.

\section{Perturbative analysis near an extremal (AdS) point of the dilaton potential}

In this appendix we will analyze here the stability properties of the perturbative dilaton potential.

We will use the domain-wall coordinate, (2.14). The field equations are given by (2.18), (2.18) and (2.19). We parametrize the potential around an AdS extremum as

$$
V=\frac{12}{\ell^{2}}-\frac{16 \xi}{3 \ell^{2}} \Phi^{2}+\mathcal{O}\left(\Phi^{3}\right)
$$

where $\Phi<<1$. Since $V^{\prime}=0$ at the critical point, there is a $A d S_{5}$ solution with

$$
A=\frac{u}{\ell} \quad, \quad \Phi=0
$$

Perturbing around the fixed point solution $A=A_{*}+\delta A, \Phi=\delta \Phi$ we obtain to linear order

$$
\frac{18}{\ell} \delta A^{\prime}=\delta \Phi^{\prime 2}-\frac{4}{\ell^{2}} \Phi^{2}=\mathcal{O}\left(\delta \Phi^{2}\right) \quad, \quad \delta \Phi^{\prime \prime}-\frac{4}{\ell} \delta \Phi^{\prime}-\frac{4 \xi}{\ell^{2}} \delta \Phi=0
$$



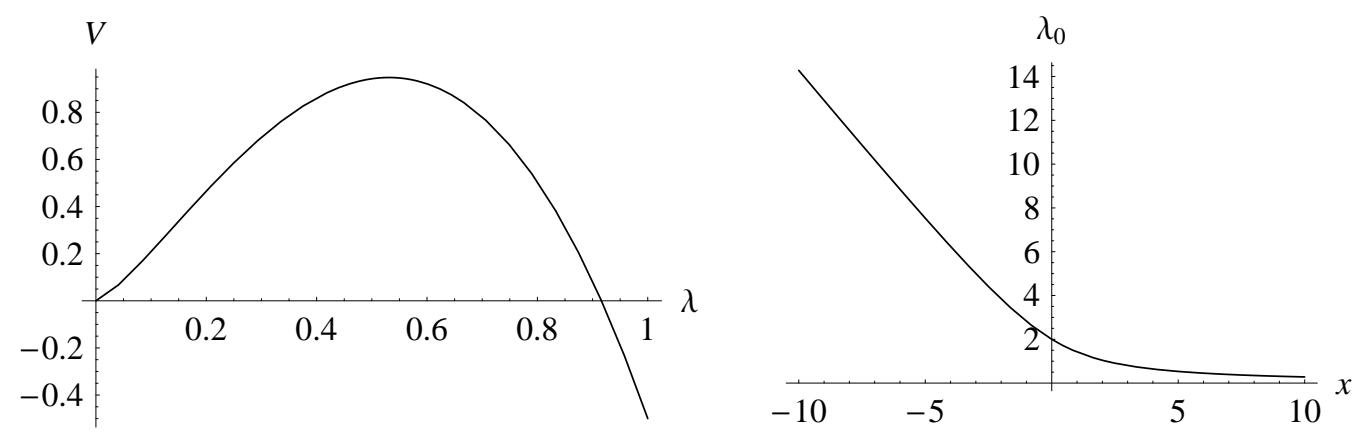

Figure 5: Left:The dilaton potential plotted as a function of the $t$ 'Hooft coupling for $x=5$. Right: The value of the $\mathrm{t}$ 'Hooft coupling at the extremum as a function of $x$.

We observe that to linear order $\delta A$ is a constant, which amounts to a renormalization of the AdS length scale $\ell$. We can therefore ignore it. The general solution of the second equation is

$$
\delta \Phi=C_{+} e^{\frac{(2+2 \sqrt{1+\xi}) u}{\ell}}+C_{-} e^{\frac{(2-2 \sqrt{1+\xi}) u}{\ell}}
$$

Changing variable to $r=L e^{u / \ell}$ we obtain to linear order in the perturbation

$$
d s^{2}=\frac{\ell^{2}}{r^{2}}\left(d r^{2}+d \vec{x}^{2}\right)+\cdots \quad, \quad \delta \Phi=C_{+}\left(\frac{r}{\ell}\right)^{(2+2 \sqrt{1+\xi})}+C_{-}\left(\frac{r}{\ell}\right)^{(2-2 \sqrt{1+\xi})}
$$

We may now analyze the potential (we assume we are in 5 dimensions and therefore $\delta c=5$ )

$$
V(\Phi)=\frac{\lambda^{\frac{4}{3}}}{\ell_{s}^{2}}\left[5-\frac{1}{2} \lambda^{2}-x \lambda\right]
$$

where

$$
x \equiv \frac{N_{f}}{N_{c}} .
$$

The potential is plotted in figure 5

It has a single extremum at $\lambda=\lambda_{0}$ with:

$$
\lambda_{0} \equiv N_{c} e^{\phi_{0}}=\frac{-7 x+\sqrt{49 x^{2}+400}}{10} .
$$

From this we obtain the value of the potential at the extremum that gives,

$$
\frac{\ell_{s}^{2}}{\ell^{2}}=\frac{\lambda_{0}^{\frac{4}{3}}}{400}\left[\frac{100+7 x^{2}-x \sqrt{49 x^{2}+400}}{400}\right]
$$

as well as the second derivative parametrized as in (C.1)

$$
\xi=\frac{5}{4}\left[\frac{400+49 x^{2}-7 x \sqrt{49 x^{2}+400}}{100+7 x^{2}-x \sqrt{49 x^{2}+400}}\right]
$$



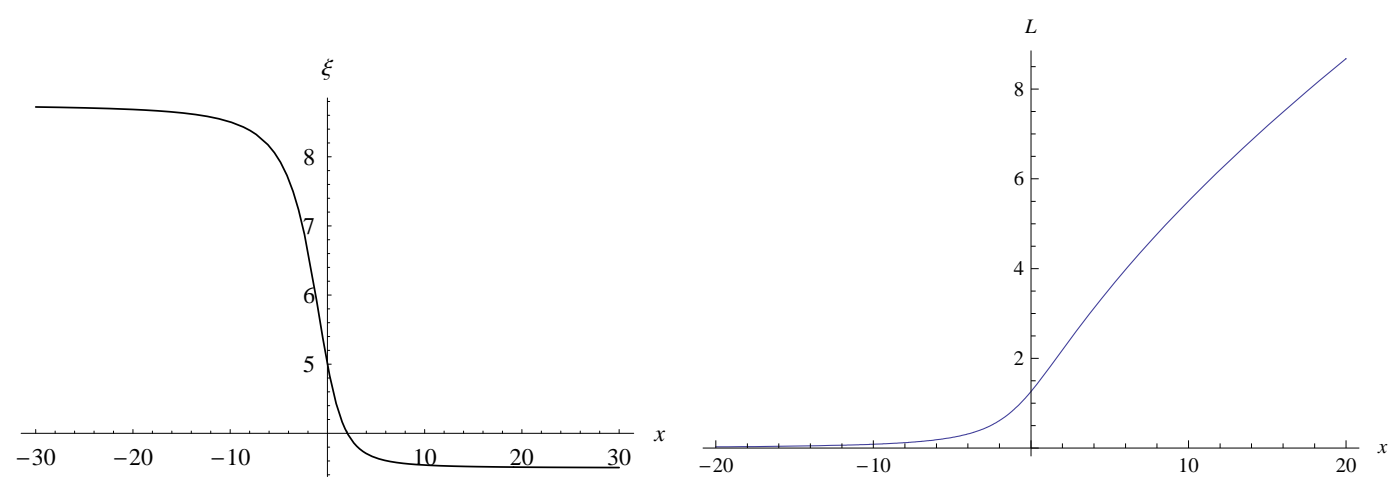

Figure 6: Left: $\xi$ plotted as a function of $x$. Right: The value of the AdS radius $L$ in units of $\ell_{s}$ as a function of $x$.

As $x$ varies between $0 \leq x \leq \infty$, the parameter $\xi$ that controls the anomalous dimension of the YM coupling constant varies as $5 \geq \xi \geq \frac{7}{2}$ as can be seen in figure 6 .

This implies that the associated dimension is $\Delta=2+2 \sqrt{1+\xi}$ and satisfies $2+3 \sqrt{2}<\Delta<2+2 \sqrt{6}$ or equivalently $6.24<\Delta<6.90$. It therefore corresponds to an irrelevant operator. This is not what we expect for QCD at weak coupling.

The AdS radius $\ell$ (in unite of $l_{s}$ ) becomes small at $x<0$, whereas it increases as $x$ becomes large as can be seen in figure 6. Therefore it becomes arbitrarily larger than the string scale for large $x$ and therefore the background will be weakly curved. Therefore, although the $t$ 'Hooft coupling at the AdS extremum is small as $x$ becomes large, the geometry is weakly curved. This is against the $\mathrm{AdS}_{5} \times \mathrm{S}^{5}$ intuition.

It should also be noted that the coefficients $N_{c}$ and $N_{f}$ appearing in the potential are multiplicatively related to the number of colors and flavors respectively. They equal them if the relations stemming from $\mathcal{N}=4$ branes still hold, but this is not in general guaranteed.

\section{Analysis of the dilaton potential with single exponential}

In the string inspired dilaton potential, the weak coupling asymptotics of the flow is governed by a single exponential in the potential. Also in the confining examples that we study in this paper, the leading term of the potential in the IR is an exponential. Therefore it is desirable to investigate the solutions of the system given by a potential of the following form (after a convenient shift in the dilaton)

$$
V(\phi)=\frac{4}{3} \epsilon e^{\alpha \phi}, \quad \epsilon= \pm 1
$$

We want to find all the solutions of the system of equations, (2.18) and (2.19) with $V$ given by (D.1). As a starting point, we classify the solutions by the behavior 
of the phase space variable $X$ which, in this case, obeys the following simple equation obtained from (3.7):

$$
\frac{d X}{d \phi}=\frac{4}{3}\left(X+\frac{1}{a}\right) \frac{\left(X^{2}-1\right)}{6 X}
$$

where we defined

$$
a=8 / 3 \alpha \text {. }
$$

The fixed points of $(\mathrm{D.2})$ are given by $X= \pm 1$ and $X=-1 / a$.

Equation (D.2) can be integrated to yield

$$
e^{\phi-\phi_{0}}=\frac{(X-1)^{\frac{3 a}{8(1+a)}}(X+1)^{\frac{3 a}{8(1-a)}}}{\left(X+\frac{1}{a}\right)^{\frac{3 a}{4\left(1-a^{2}\right)}}} .
$$

For the special case of $a= \pm 1$ we obtain instead

$$
\log \frac{X-1}{X+1}-\frac{2}{X \pm 1}=\frac{16}{3}\left(\phi-\phi_{0}\right)
$$

Now, the solutions of the system are given by the fixed point solutions of (D.2) and the solutions that flow between these fixed points. We first list the fixed point solutions:

\section{D.1 The $X=1$ fixed point}

This amounts to solving

$$
\phi^{\prime}=3 A^{\prime} \quad, \quad \phi^{\prime \prime}+\frac{4}{3} \phi^{\prime 2}=0 \quad, \quad e^{\alpha \phi} \rightarrow 0
$$

with solution

$$
e^{\phi}=C\left(u_{0}-u\right)^{\frac{3}{4}}
$$

We take the range of $u$ as $u \in\left(-\infty, u_{0}\right)$. If $\alpha>0$ this solution is valid in the immediate neighborhood of $u=u_{0}$. If $\alpha<0$ it is valid in the neighborhood $u \rightarrow \infty$. We also find

$$
e^{A}=\tilde{C}\left(u_{0}-u\right)^{\frac{1}{4}}
$$

and for the Poincaré coordinate

$$
r-r_{0}=\frac{4}{3 \tilde{C}}\left(u_{0}-u\right)^{\frac{3}{4}} \quad, \quad b(r)=\left(\frac{3 \tilde{C}^{4}}{4}\right)^{\frac{1}{3}}\left(r-r_{0}\right)^{\frac{1}{3}} \quad, \quad e^{\phi}=\frac{3}{4} C \tilde{C}\left(r-r_{0}\right)
$$

This solution is valid near $r=r_{0}$.

\section{D.2 The $X=-1$ fixed point}

This amounts to solving

$$
\phi^{\prime}=-3 A^{\prime} \quad, \quad \phi^{\prime \prime}-\frac{4}{3} \phi^{2}=0 \quad, \quad e^{\alpha \phi} \rightarrow 0
$$

It is related to the $X=1$ solution by $\phi \rightarrow-\phi$. 


\section{D.3 The $X=-\frac{1}{a}$ fixed point}

It exists if

(a) $\epsilon=1$ and $|a|>1$

(b) $\epsilon=-1$ and $|a|<1$

(c) $\epsilon= \pm 1$ and $|a|=1$. In this case it merges with the $X= \pm 1$ fixed point solutions.

The solution is

$$
e^{\phi}=\left(\frac{C}{u_{0}-u}\right)^{-\frac{3 a}{4}} \quad, \quad e^{A}=\tilde{C}\left(u_{0}-u\right)^{\frac{a^{2}}{4}} \quad, \quad C=\frac{3 a}{4} \sqrt{\epsilon\left(a^{2}-1\right)}
$$

In the Poincaré coordinate,

$$
\begin{gathered}
r-r_{0}=\frac{4}{\left(4-a^{2}\right) \tilde{C}}\left(u_{0}-u\right)^{\frac{4-a^{2}}{4}}, e^{A}(r)=\tilde{C}\left(\frac{\left(4-a^{2}\right) \tilde{C}}{4}\right)^{\frac{a^{2}}{4-a^{2}}}\left(r-r_{0}\right)^{\frac{a^{2}}{4-a^{2}}} \\
e^{\phi}=C^{-\frac{3 a}{4}}\left(\frac{\left(4-a^{2}\right) \tilde{C}}{4}\right)^{\frac{3 a}{4-a^{2}}}\left(r-r_{0}\right)^{\frac{3 a}{4-a^{2}}}
\end{gathered}
$$

\section{D.4 Flow solutions}

More general solutions are given by the flows between the fixed points of $X$. The nature of these flows are determined by the stability properties of the fixed points that we depict in fig.(17) for the case $a<0$. A cross denotes an unstable fixed point and a point denotes a stable one. The arrows between these points depict the direction of the flow in an appropriate radial variable that we define below. The boundaries of the phase space are given by $X= \pm \infty$ and $X=0$. In particular the $X<0$ and the $X>0$ solutions are disconnected. The case $a>0$ can be obtained from fig.(7) by utilizing the symmetry of (D.2) under $X \rightarrow-X, \phi \rightarrow-\phi$ and $a \rightarrow-a$.

The system can be solved analytically in the following radial variable, $t$ :

$$
\frac{d}{d u}=e^{\frac{\alpha_{s}}{2} \phi} \frac{d}{d t}
$$

We describe the solution for the case $\epsilon=+1$ which is the interesting case for us. In this case the range of the phase space variable is given by $-1<X<1$. This range is divided into two flow parts by the presence of the fixed point at $X=-1 / a$. Let us take $a>1(a<1$ is given by a simple replacement of the hyperbolic functions below with the triangular ones).

D.4.1 The solution: $-1<X<-1 / a$

Let us define,

$$
\bar{t}=\frac{2 \sqrt{a^{2}-1}}{3 a}\left(t-t_{0}\right)
$$


A

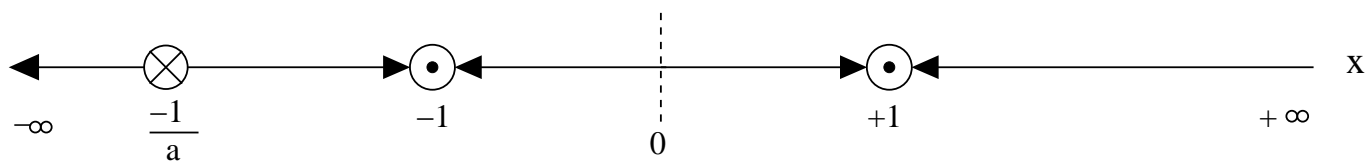

$\mathrm{B}$

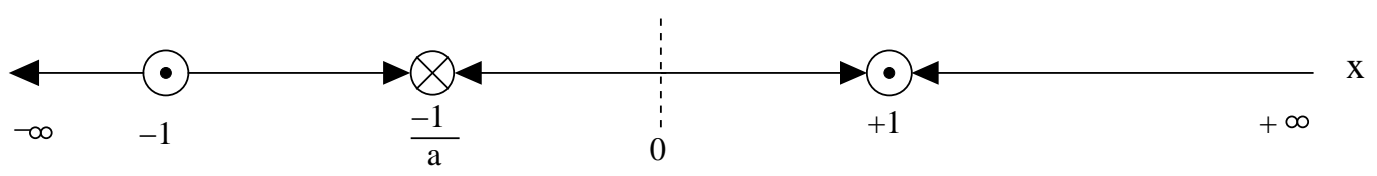

Figure 7: The stability properties of the fixed points. Case A is for $0<a<1$ and case B corresponds to $a>1$. The cases where $a<0$ can be obtained from the above figures by using the reflection symmetry in $X$.

The solution is given by,

$$
\phi=\phi_{0}+\frac{3 a}{4}\left(\frac{1}{a+1} \log \cosh (\bar{t})-\frac{1}{a-1} \log \sinh (\bar{t})\right),
$$

and

$$
A=A_{0}+\frac{a}{4}\left(\frac{1}{a+1} \log \cosh (\bar{t})+\frac{1}{a-1} \log \sinh (\bar{t})\right) .
$$

The phase space variable as a function of $\bar{t}$ is given by,

$$
X(\bar{t})=\frac{\frac{a-1}{a+1} \tanh ^{2}(\bar{t})-1}{\frac{a-1}{a+1} \tanh ^{2}(\bar{t})+1}
$$

In these equations, we choose $\bar{t}>0$ that corresponds to the range $t_{0}<t<\infty$. This solution corresponds to the flow from the fixed point $X=-1$ at $t=t_{0}$ to the fixed point $X=-1 / a$ at $t=\infty$.

The asymptotics of this solution are as follows. As $t \rightarrow t_{0},(X \rightarrow-1)$,

$$
\begin{aligned}
\lambda & \rightarrow\left(t-t_{0}\right)^{\frac{3 a}{4(a-1)}} \\
d s^{2} & \rightarrow\left(t-t_{0}\right)^{\frac{a}{2(a-1)}} d x^{2}+\left(t-t_{0}\right)^{\frac{2}{a-1}} d t^{2}
\end{aligned}
$$

Thus the space shrinks to a point as one approaches to the $X=-1$ fixed point. As $t \rightarrow \infty,(X \rightarrow-1 / a)$,

$$
\begin{aligned}
\lambda & \rightarrow e^{-\frac{t}{\sqrt{a^{2}-1}}} \\
d s^{2} & \rightarrow \frac{1}{r^{2}}\left(d x^{2}+r^{-\frac{8}{a^{2}}} d r^{2}\right)
\end{aligned}
$$


where in the last equation we changed the variable as $r=\exp \left(-a t / \sqrt{a^{2}-1}\right)$, hence $r \rightarrow 0$ in the limit. We observe from the asymptotics of the metric, that the space becomes AdS only when $a$ is taken to $\infty$. This nicely parallels the fact that as $a \rightarrow \infty$ the fixed point approaches to $X \rightarrow 0$ and, as our primary example in this paper suggests, one has an asymptotically AdS space in the $X \rightarrow 0$ limit.

\section{D.4.2 The solution: $-1 / a<X<+1$}

The solution is given by,

$$
\phi=\phi_{0}+\frac{3 a}{4}\left(-\frac{1}{a-1} \log \cosh (\bar{t})+\frac{1}{a+1} \log \sinh (\bar{t})\right),
$$

and

$$
A=A_{0}+\frac{a}{4}\left(\frac{1}{a-1} \log \cosh (\bar{t})+\frac{1}{a+1} \log \sinh (\bar{t})\right) .
$$

The phase space variable as a function of $\bar{t}$ is given by,

$$
X(\bar{t})=\frac{-\frac{a+1}{a-1} \tanh ^{2}(\bar{t})+1}{\frac{a+1}{a-1} \tanh ^{2}(\bar{t})+1} .
$$

This solution is valid in the whole range $-1 / a<X<+1$ (for $a>1$ ) and describes the two flow solutions: one from $X=0$ to $X=-1 / a$ and from $X=-1 / a$ and one from $X=0$ to $X=1$.

The interesting asymptotics are $t \rightarrow \infty,(X \rightarrow-1 / a)$ and $t \rightarrow t_{1}$ where,

$$
t_{1}=t_{0}+\frac{3 a}{2 \sqrt{a^{2}-1}} \tanh ^{-1} \sqrt{\frac{a-1}{a+1}} .
$$

In the first case, as $X \rightarrow-1 / a$, one obtains the same asymptotics as in (D.21) and (D.22). In the second case, as $X \rightarrow 0$, one finds,

$$
\begin{gathered}
\lambda \rightarrow \text { const. } \\
d s^{2} \rightarrow d x^{2}+d t^{2} .
\end{gathered}
$$

\section{E. $\beta$-function with an exponential tail}

In this appendix we provide another example of an exact $\beta$-function and the associated holographic geometry. We choose the $\beta$-function here as:

$$
\beta=-b_{0} \lambda^{2} e^{-c_{0} \lambda}
$$

In perturbation theory this describes an asymptotically free theory. In the IR it reaches a fixed point but in way which is different from the previous example. In particular the fixed point is at infinite coupling. 


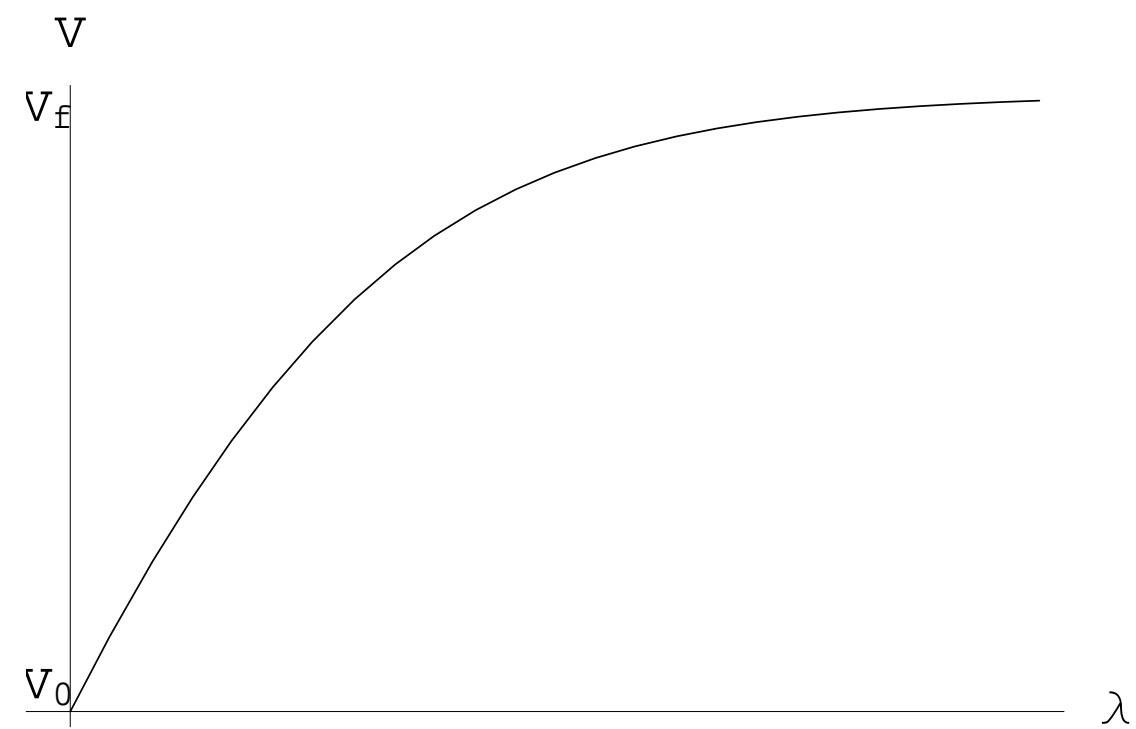

Figure 8: Scalar potential as a function of $\lambda$ for the exponential type running. We set $V_{0}=b_{0}=c_{0}=1$ for illustrative purposes.

The potential that follows from this $\beta$-function is obtained from (3.8) as,

$$
V=V_{0}\left(1-\frac{b_{0}^{2}}{9} \lambda^{2} e^{-2 c_{0} \lambda}\right) e^{-\frac{8 b_{0}}{9 c_{0}}\left(e^{-c_{0} \lambda}-1\right)},
$$

The plot of the potential is shown in fig. 8. The RG flow is from the Gaussian fixed point in the UV to an IR fixed point at $\lambda=\infty$ in the IR. The corresponding UV geometry is an asymptotically AdS space with radius given by (4.12) as before. The corresponding far IR geometry is also AdS as expected from the fact that $V$ in (E.2) approaches to a constant as $\lambda \rightarrow \infty$ :

$$
V \rightarrow V_{f}=V_{0} e^{\frac{8 b_{0}}{9 c_{0}}}
$$

The radius of the AdS in the IR is given by,

$$
\ell_{I R}=2 \sqrt{\frac{3}{V_{f}}} .
$$

The running of the 't Hooft coupling is determined by the following differential equation that follows from (3.5):

$$
\lambda^{\prime}=\frac{\sqrt{3 V_{f}}}{6} b_{0} \lambda^{2} e^{-c_{0} \lambda} e^{-\frac{4 b_{0}}{9 c_{0}}\left(e^{-c_{0} \lambda}-1\right)} .
$$

We display the numerical solution to this equation in figure 9. From this figure it is clear that the coupling constant diverges in the IR. One can calculate the scalar 


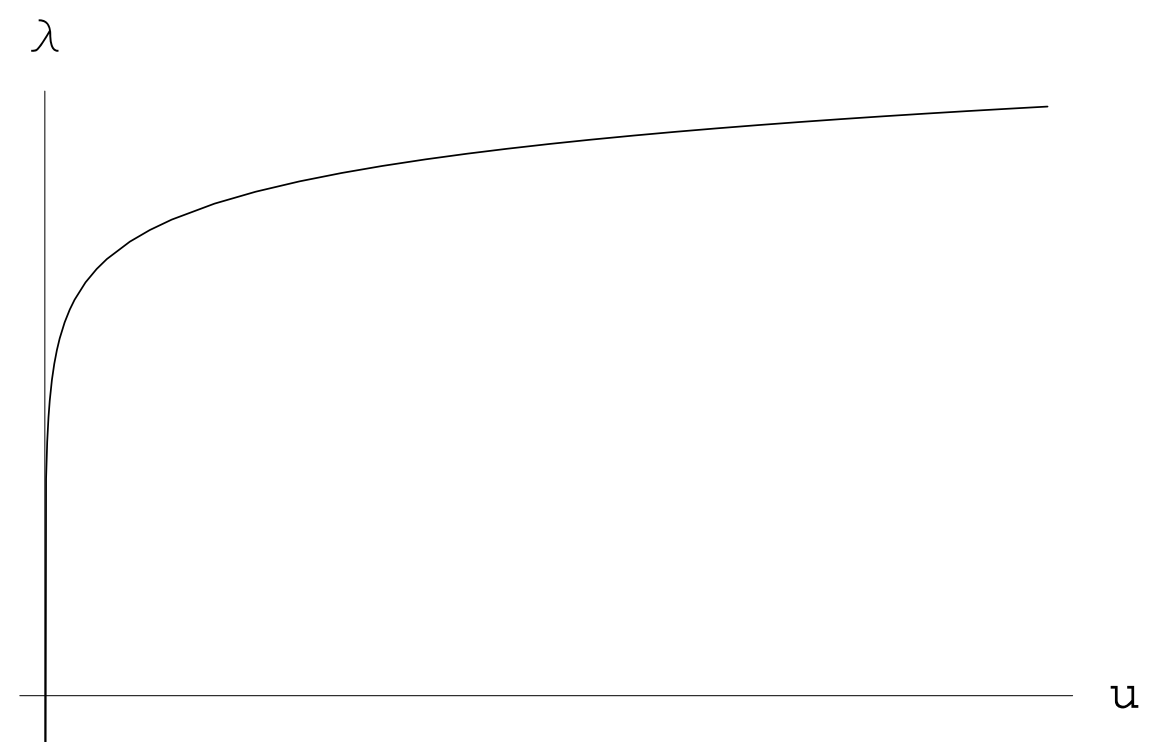

Figure 9: Running of the coupling constant in the exponential case. We have set $V_{0}=$ $b_{0}=c_{0}=1$.

invariants of the geometry. The Ricci scalar is given by (3.9) and $R=-3 / 2 E$. In the Einstein frame, one obtains the IR limit value of $R$ as,

$$
R=-\frac{15}{4} V_{f}
$$

where $V_{f}$ is given by (E.3). Note that the function $X$ in (3.4) limits to zero both in the UV and the IR. This is in accord with the fact that the scalar potential goes over to constant values both in the UV and the IR. The 5D geometry is of the form of a Minkowski domain wall that interpolates between two AdS geometries.

The details of the approach towards the IR AdS geometry can be studied as in section 5.2. One determines the following subleading behavior near the IR fixed point:

$$
d s^{2}=\frac{l_{I R}^{2}}{r^{2}}\left(1-\frac{4}{9} \frac{1}{r \Lambda} \frac{1}{\log \log (r \Lambda)}\right)^{-2}\left(d x^{2}+d r^{2}\right)
$$

as $r \rightarrow \infty$.

\section{References}

[1] G. 't Hooft, "A planar diagram theory for strong interactions" Nucl. Phys. B 72 (1974) 461.

[2] J. M. Maldacena, "The large $N$ limit of superconformal field theories and supergravity," Adv. Theor. Math. Phys. 2, 231 (1998) [Int. J. Theor. Phys. 38, 1113 (1999)] [ArXiv:hep-th/9711200]; 
S. S. Gubser, I. R. Klebanov and A. M. Polyakov, "Gauge theory correlators from non-critical string theory," Phys. Lett. B 428, 105 (1998) [ArXiv:hep-th/9802109];

E. Witten, "Anti-de Sitter space and holography", Adv. Theor. Math. Phys. 2, 253 (1998); ArXiv:hep-th/9802150.

[3] E. Witten, "Anti-de Sitter space, thermal phase transition, and confinement in gauge theories," Adv. Theor. Math. Phys. 2 (1998) 505 [ArXiv:hep-th/9803131].

[4] A. H. Chamseddine and M. S. Volkov, "Non-Abelian BPS monopoles in $N=4$ gauged supergravity," Phys. Rev. Lett. 79 (1997) 3343 [ArXiv:hep-th/9707176].

[5] J. M. Maldacena and C. Nunez, "Towards the large $N$ limit of pure $N=1$ super Yang Mills," Phys. Rev. Lett. 86 (2001) 588 [ArXiv:hep-th/0008001.

[6] I. R. Klebanov and M. J. Strassler, "Supergravity and a confining gauge theory: Duality cascades and $\chi S B$-resolution of naked singularities," JHEP 0008 (2000) 052 [ArXiv:hep-th/0007191].

[7] C. Csaki, H. Ooguri, Y. Oz and J. Terning, "Glueball mass spectrum from supergravity," JHEP 9901 (1999) 017 [ArXiv:hep-th/9806021];

R. C. Brower, "AdS/CFT duality and the glueball spectrum," Int. J. Mod. Phys. A 16S1C (2001) 1005;

H. Boschi-Filho and N. R. F. Braga, "QCD / string holographic mapping and glueball mass spectrum,” Eur. Phys. J. C 32, 529 (2004) [ArXiv:hep-th/0209080] "Gauge / string duality and scalar glueball mass ratios," JHEP 0305, 009 (2003) [ArXiv:hep-th/0212207];

E. Caceres, "A Brief Review Of Glueball Masses From Gauge / Gravity Duality," వ Phys. Conf. Ser. 24 (2005) 111.

[8] F. Bigazzi, R. Casero, A. L. Cotrone, E. Kiritsis and A. Paredes, "Non-critical holography and four-dimensional CFT's with fundamentals," JHEP 0510, 012 (2005) [ArXiv:hep-th/0505140].

[9] M. Henningson, K. Skenderis, The Holographic Weyl anomaly, JHEP 07 (1998) 023, [ArXiv:hep-th/9806087.

[10] S. Kuperstein and J. Sonnenschein, "Non-critical supergravity $(d>1)$ and holography," JHEP 0407 (2004) 049 [ArXiv:hep-th/0403254];

S. Kuperstein and J. Sonnenschein, "Non-critical, near extremal AdS6 background as a holographic laboratory of four dimensional YM theory," JHEP 0411 (2004) 026 [ArXiv:hep-th/0411009];

R. Casero, A. Paredes and J. Sonnenschein, "Fundamental matter, meson spectroscopy and non-critical string / gauge duality," JHEP 0601 (2006) 127 [ArXiv:hep-th/0510110].

[11] I. R. Klebanov and J. M. Maldacena, "Superconformal gauge theories and non-critical superstrings," Int. J. Mod. Phys. A 19 (2004) 5003 [ArXiv:hep-th/0409133. 
[12] C. Csaki and M. Reece, "Toward a systematic holographic QCD: A braneless approach,” JHEP 0705 (2007) 062 [ArXiv:hep-ph/0608266].

[13] A. Fotopoulos, Semiclassical description of D-branes in $S L(2) / U(1)$ gauged $W Z W$ model, Class. Quant. Grav. 20, S465 (2003), [ArXiv:hep-th/0304015].

[14] A. Fotopoulos, V. Niarchos and N. Prezas, "D-branes and extended characters in $S L(2, R) / U(1)$," Nucl. Phys. B 710 (2005) 309 [ArXiv:hep-th/0406017]; "D-branes and SQCD in non-critical superstring theory," JHEP 0510 (2005) 081 [ArXiv:hep-th/0504010].

[15] S. K. Ashok, S. Murthy and J. Troost, "D-branes in non-critical superstrings and minimal super Yang-Mills in various dimensions," Nucl. Phys. B 749 (2006) 172 [ArXiv:hep-th/0504079];

S. Murthy and J. Troost, "D-branes in non-critical superstrings and duality in $N=1$ gauge theories with flavor," JHEP 0610 (2006) 019 [ArXiv:hep-th/0606203.

[16] D. Israel and V. Niarchos, "Orientifolds in $N=2$ Liouville theory and its mirror," ArXiv:hep-th/0703151]; "Tree-level stability without spacetime fermions: Novel examples in string theory," JHEP 0707 (2007) 065 [ArXiv:0705.2140] [hep-th].

[17] J. Polchinski and M. J. Strassler, "Hard scattering and gauge/string duality," Phys. Rev. Lett. 88 (2002) 031601 [ArXiv:hep-th/0109174]; "Hard scattering and gauge/string duality," Phys. Rev. Lett. 88 (2002) 031601 [ArXiv:hep-th/0109174].

[18] J. Erlich, E. Katz, D. T. Son and M. A. Stephanov, "QCD and a holographic model of hadrons," Phys. Rev. Lett. 95, 261602 (2005) [arXiv:hep-ph/0501128].

[19] L. Da Rold and A. Pomarol, "Chiral symmetry breaking from five dimensional spaces," Nucl. Phys. B 721, 79 (2005) [ArXiv:hep-ph/0501218.

[20] U. Gursoy, E. Kiritsis and F. Nitti, "Exploring improved holographic theories for QCD: Part II," [ArXiv:0707.1349] [hep-th]

[21] M. A. Shifman, A. I. Vainshtein and V. I. Zakharov, "QCD And Resonance Physics. Sum Rules," Nucl. Phys. B 147 (1979) 385.

[22] S. S. Gubser, "Curvature singularities: The good, the bad, and the naked," Adv. Theor. Math. Phys. 4 (2002) 679 [ArXiv:hep-th/0002160].

[23] T. Sakai and S. Sugimoto, "Low energy hadron physics in holographic QCD," Prog. Theor. Phys. 113 (2005) 843 [ArXiv:hep-th/0412141].

[24] R. Casero, E. Kiritsis and A. Paredes, "Chiral symmetry breaking as open string tachyon condensation," [ArXiv:hep-th/0702155.

[25] A. L. Cotrone, "On the YM and QCD spectra from five dimensional strings," [ArXiv:0707.1483][hep-th]. 
[26] E. Witten, "Instantons, The Quark Model, And The 1/N Expansion," Nucl. Phys. B 149 (1979) 285.

[27] E. Witten, "Current Algebra Theorems For The U(1) Goldstone Boson," Nucl. Phys. B 156, 269 (1979);

G. Veneziano, “U(1) Without Instantons," Nucl. Phys. B 159, 213 (1979).

[28] E. Witten, "Theta dependence in the large $N$ limit of four-dimensional gauge theories," Phys. Rev. Lett. 81, 2862 (1998) [ArXiv:hep-th/9807109].

[29] K. Skenderis and P. K. Townsend, "Gravitational stability and renormalization-group flow," Phys. Lett. B 468 (1999) 46 [ArXiv:hep-th/9909070];

D. Z. Freedman, C. Nunez, M. Schnabl and K. Skenderis, "Fake supergravity and domain wall stability," Phys. Rev. D 69 (2004) 104027 [ArXiv:hep-th/0312055 K. Skenderis and P. K. Townsend, "Hidden supersymmetry of domain walls and cosmologies," Phys. Rev. Lett. 96 (2006) 191301 [ArXiv:hep-th/0602260];

"Hamilton-Jacobi for domain walls and cosmologies," Phys. Rev. D 74 (2006) 125008 [ArXiv:hep-th/0609056].

[30] S. R. Das and S. P. Trivedi, "Three brane action and the correspondence between N = 4 Yang Mills theory and anti de Sitter space," Phys. Lett. B 445 (1998) 142 [ArXiv:hep-th/9804149];

S. Ferrara, M. A. Lledo and A. Zaffaroni, "Born-Infeld corrections to D3 brane action in $A d S(5) x S(5)$ and $N=4, d=4$ primary superfields," Phys. Rev. D 58 (1998) 105029 [ArXiv:hep-th/9805082.

[31] J. Polchinski and M. J. Strassler, "The string dual of a confining four-dimensional gauge theory," [ArXiv:hep-th/0003136].

[32] R. Marotta and F. Sannino, " $N=1$ super Yang-Mills renormalization schemes for fractional branes," Phys. Lett. B 545 (2002) 162 [ArXiv:hep-th/0207163].

[33] A. W. Peet and J. Polchinski, "UV/IR relations in AdS dynamics," Phys. Rev. D 59 (1999) 065011 [ArXiv:hep-th/9809022.

[34] J. M. Maldacena, "Wilson loops in large N field theories," Phys. Rev. Lett. 80 (1998) 4859 ArXiv:hep-th/9803002.

[35] S. J. Rey and J. T. Yee, "Macroscopic strings as heavy quarks in large $N$ gauge theory and anti-de Sitter supergravity," Eur. Phys. J. C 22 (2001) 379 [ArXiv:hep-th/9803001].

[36] L. Girardello, M. Petrini, M. Porrati and A. Zaffaroni, "Novel local CFT and exact results on perturbations of $N=4$ super Yang-Mills from AdS dynamics," JHEP 9812 (1998) 022 ArXiv:hep-th/9810126;

D. Z. Freedman, S. S. Gubser, K. Pilch and N. P. Warner, "Renormalization group flows from holography supersymmetry and a c-theorem," Adv. Theor. Math. Phys. 3, 363 (1999) [ArXiv:hep-th/9904017. 
[37] M. Bianchi, M. Prisco and W. Muck, "New results on holographic three-point functions," JHEP 0311 (2003) 052 [ArXiv:hep-th/0310129].

[38] L. Kofman, J. Martin and M. Peloso, "Exact identification of the radion and its coupling to the observable sector," Phys. Rev. D 70 (2004) 085015

[ArXiv:hep-ph/0401189].

[39] C. Csaki, J. Erlich, T. J. Hollowood and Y. Shirman, "Universal aspects of gravity localized on thick branes," Nucl. Phys. B 581, 309 (2000) [ArXiv:hep-th/0001033;

I. Papadimitriou and K. Skenderis, "Correlation functions in holographic RG flows," JHEP 0410 (2004) 075 [ArXiv:hep-th/0407071.

[40] E. Kiritsis and F. Nitti, "On massless $4 D$ Gravitons from 5D Asymptotically AdS Space-times," [ArXiv:hep-th/0611344].

[41] S. Narison, "Masses, decays and mixings of gluonia in QCD," Nucl. Phys. B 509 (1998) 312 [ArXiv:hep-ph/9612457].

[42] A. M. Polyakov, "The wall of the cave," Int. J. Mod. Phys. A 14 (1999) 645 [ArXiv:hep-th/9809057].

[43] C. Angelantonj and A. Armoni, "Non-tachyonic type $0 B$ orientifolds, non-supersymmetric gauge theories and cosmological RG flow," Nucl. Phys. B 578 (2000) 239 [ArXiv:hep-th/9912257;

"RG flow, Wilson loops and the dilaton tadpole," Phys. Lett. B 482 (2000) 329

[ArXiv:hep-th/0003050]. 